\title{
Overcomplete Discrete Wavelet Transforms with Rational Dilation Factors
}

\author{
İlker Bayram, Student Member, IEEE and Ivan W. Selesnick, Senior Member, IEEE
}

\begin{abstract}
This paper develops an overcomplete discrete wavelet transform (DWT) based on rational dilation factors for discrete-time signals. The proposed overcomplete rational DWT is implemented using selfinverting FIR filter banks, is approximately shift-invariant, and can provide a dense sampling of the timefrequency plane. A straightforward algorithm is described for the construction of minimal-length perfect reconstruction filters with a specified number of vanishing moments; whereas, in the non-redundant rational case, no such algorithm is available. The algorithm is based on matrix spectral factorization. The analysis/synthesis functions (discrete-time wavelets) can be very smooth and can be designed to closely approximate the derivatives of the Gaussian function.
\end{abstract}

\section{Index Terms}

Wavelet transforms, frame, filter bank, rational dilation factor, matrix spectral factorization.

\section{INTRODUCTION}

Overcomplete transforms, or 'frames', have become a well recognized tool in signal processing [28]. During the last decade numerous overcomplete wavelet transforms (wavelet frames) have been designed and utilized for applications. Most of these frames are dyadic wavelet transforms; the resolution is doubled from each scale to the next scale. This paper describes the construction of wavelet frames for discrete-time signals where the resolution is increased more gradually from scale to scale. Here, the dilation factor is a rational number between one and two, and we have a 'rational' wavelet transform.

Manuscript received January 23, 2008; Revised July 17, 2008.

İ. Bayram and I. W. Selesnick are with the Department of Electrical and Computer Engineering, Polytechnic Institute of New York University, Brooklyn, NY 11201. Phone: (718) 260-3416, Fax: (718) 260-3906, E-mail: ibayra01@ @tudents.poly.edu, selesi@poly.edu 
Although the theory [3], [4], [7], [26], implementation [29], design algorithms [9], [2], and application [6], [8] of critically-sampled rational discrete wavelet transforms (DWTs) are known, the overcomplete case has not been investigated as far as we are aware. This paper examines the properties and construction of the overcomplete rational DWT. It is found that the overcomplete rational DWT provides several advantages compared to the critically-sampled rational DWT. First, minimal-length perfect reconstruction filters with a specified number of vanishing moments can be straightforwardly constructed. (For criticallysampled rational DWTs, only a few solutions with more than a single vanishing moment are known [5].) Second, the overcomplete rational DWT can be approximately shift-invariant. (For the criticallysampled rational DWT the lack of shift-invariance is even more severe than is so for the critically-sampled dyadic DWT.) Third, for the same impulse response length, the analysis/synthesis functions (discrete-time wavelets) can be much smoother in the overcomplete case.

One motivation for developing rational wavelet transforms is the higher frequency resolution that can be achieved, compared to the dyadic and $M$-band wavelet transforms $(M \in \mathbb{Z})$. However, the improved frequency resolution is not emphasized in this paper (although the Q factors for an ideal rational DWT are calculated in Section VIII).

Although we develop rational wavelet frames strictly for discrete-time signals, we use the terms 'dilation factor' and 'wavelet' which are often affiliated with an underlying multiresolution analysis (MRA) of $L_{2}(\mathbb{R})$. In fact, an MRA with rational dilation factor can be constructed similar to the integer dilation case [3]. Also, a fast algorithm for the computation of the expansion coefficients is equivalent to an iterated FB with rational sampling factors [4]. However, a rational MRA does not allow basis functions to be compactly-supported, and hence the FBs induced cannot be implemented with FIR filters. Nevertheless, Blu shows that when iterated, FIR FBs with rational sampling factors can be made to yield smooth functions [7]. Even though these functions do not form an MRA, iterated FBs with rational sampling factors can yield well behaved bases or frames for $l_{2}(\mathbb{Z})$. By 'wavelets' we refer to the discrete-time analysis/synthesis functions generated by the iterated FB. We say the 'dilation factor' is rational because the low-pass channel of the FB is a rate-changer with a rational rate conversion factor.

For convenience of notation and clarity, this paper emphasizes the design of rational wavelet transforms for which the dilation factor is $3 / 2$. However, the ideas and constructions carry over to other rational dilation factors as well. (In some cases it may be desirable to use different dilation factors at different scales: a critically-sampled orthonormal Haar-like transform is developed in [20] in which the dilation factors follow the Fibonacci sequence, such that in the limit the dilation factor is the Golden Ratio.) Throughout the paper, it is assumed that all impulse responses are real-valued. Also, throughout the 


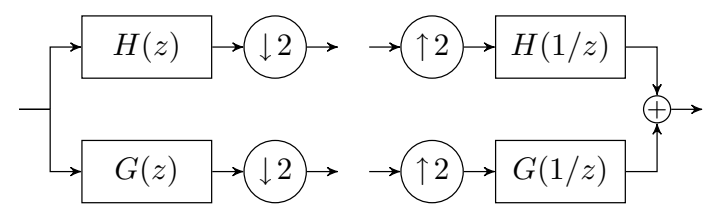

Fig. 1. A critically-sampled dyadic discrete-time filter bank. The dilation factor is 2 .

paper, the synthesis filters will always be the time-reversed versions of the analysis filters; therefore, the overcomplete transforms developed in this paper are tight-frames. When unambiguous, we write $H(\omega)$ for $H\left(e^{j \omega}\right)$. Some of the results presented here appear in an earlier conference publication [5].

\section{A. Time-Frequency Sampling Lattice}

It is informative to consider the way in which the proposed overcomplete rational wavelet transform (WT) samples the time-frequency plane. This motivates the development of the transform and places it in context with some other overcomplete wavelet transforms.

Consider first, the critically-sampled dyadic discrete wavelet transform, implemented via iteration of the low-pass/high-pass filter bank in Fig. 1. The time-frequency lattice is illustrated in the top panel of Fig. 2. The scales are logarithmically distributed in frequency and each scale is uniformly sampled in time (each scale is sampled at half the rate of the next finer scale). For discussion of this wavelet time-frequency lattice see [45, p. 273] [16, p. 9]. For background on the DWT, see for example [45, Chp. 3].

Overcomplete wavelet transforms are characterized by denser time-frequency lattices. For example, the undecimated discrete wavelet transform (UDWT, also called the 'algorithme à trous') [25], samples each scale at the same full rate as the input signal; the time-frequency lattice of the UDWT is illustrated in [28, Fig. 2]. The UDWT is $(J+1)$-times overcomplete, where $J$ is the number of scales (the depth of the iterated filter bank). Therefore, although effective and easily implemented, the UDWT can be overly overcomplete - more than necessary and more than can be afforded in some applications. This type of time-frequency sampling scheme deviates from the wavelet schema - one expects that each scale be sampled at a lower rate than the next finer scale, in order to achieve an efficient distribution of sampling points in the time-frequency plane.

An alternative overcomplete DWT that maintains the wavelet time-frequency sampling schema is the 'double-density' discrete wavelet transform, which can be implemented using an iterated overcomplete 

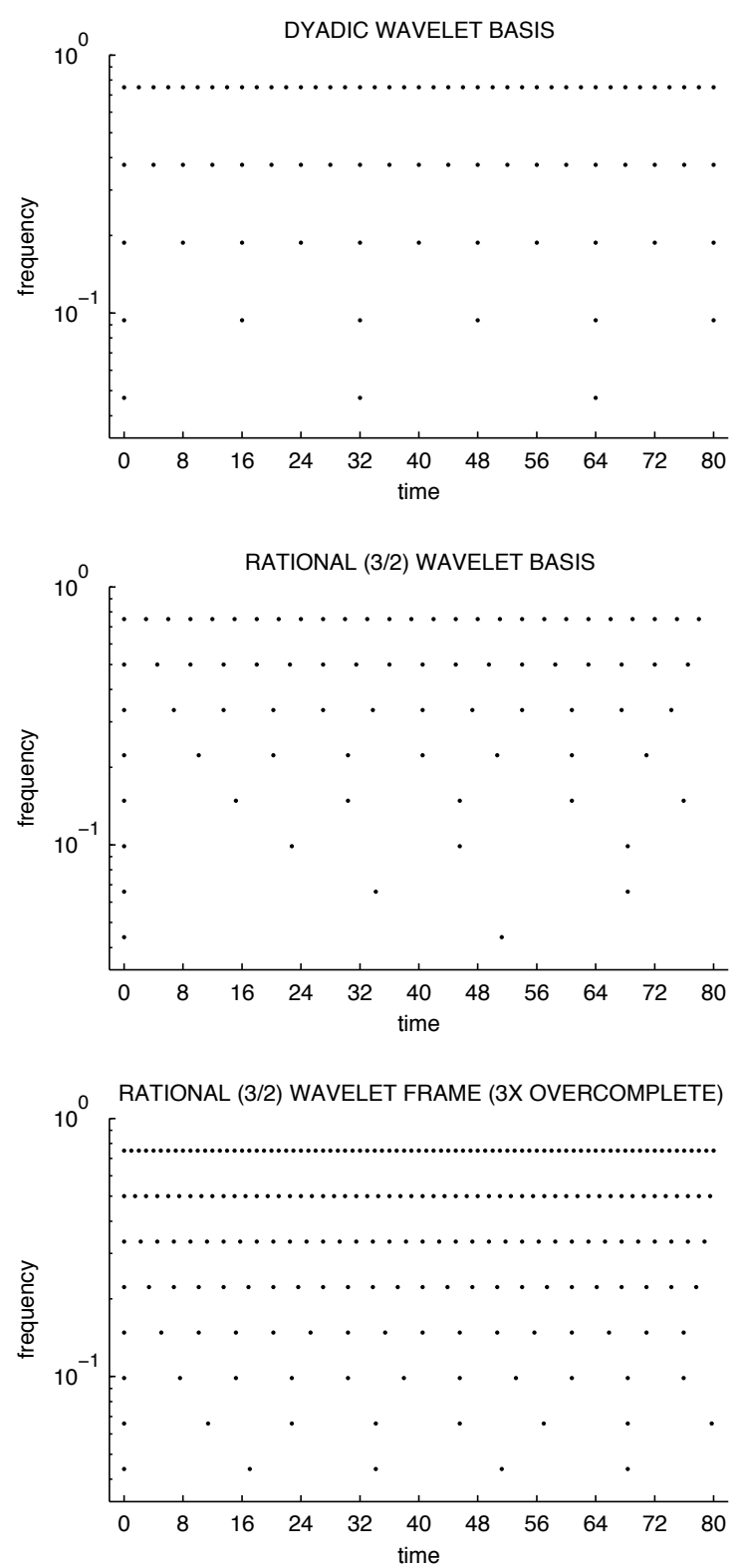

Fig. 2. The time-frequency sampling lattice for three different wavelet transforms (WTs). Top panel: dyadic WT implemented using the filter bank in Fig. 1. Middle panel: critically-sampled rational WT implemented using the filter bank in Fig. 3. Bottom panel: the overcomplete rational WT implemented using the filter bank in Fig. 7. 


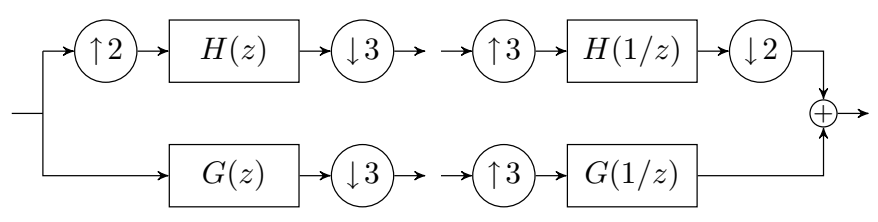

Fig. 3. A critically-sampled discrete-time filter bank with rational dilation factor. The dilation factor is $3 / 2$.

filter bank [38] or the Laplacian pyramid [10]. The idea of the double-density DWT is to twice oversample each scale of the dyadic wavelet lattice in order to create an overcomplete DWT that maintains the wavelet time-frequency sampling schema and which can be implemented for discrete-time data using an invertible filter bank. The double-density DWT is two-times overcomplete.

To create a three-times overcomplete DWT by thrice oversampling each scale of a critically-sampled wavelet transform, one should start with the time-frequency lattice illustrated in the middle panel of Fig. 2. This is the lattice of the critically-sampled rational DWT with dilation factor $3 / 2$, implemented via iteration of the low-pass/high-pass filter bank illustrated in Fig. 3. In this lattice, the finest scale is sampled at $1 / 3$ the full rate of the input signal, and each scale is sampled at $2 / 3$ the rate of the previous scale. The scales are more closely spaced in frequency than is so for the dyadic lattice. Thrice oversampling each scale of this lattice leads to the lattice illustrated in the bottom panel of Fig. 2, the finest scale of which is sampled at the same rate as the input signal.

This approach generalizes the double-density DWT to higher (more than 2) redundancy. To create a $d$-times overcomplete DWT one can similarly start with the time-frequency lattice of a critically-sampled rational WT with dilation factor $d /(d-1)$, and over-sample each scale by $d$. The resulting discrete-time wavelet transform will be approximately shift-invariant (more so for higher $d$ ), yet unlike the UDWT, it maintains the wavelet time-frequency sampling schema. In comparison with the time-frequency lattice of the critically-sampled dyadic DWT, the new transform is denser in both time and frequency, not just denser in time like the UDWT. The invertible filter bank implementation with FIR filters of such a transform, and the design of such filter banks, is the main contribution of this paper.

In earlier work [39], a three-times overcomplete DWT, called the 'higher-density' DWT, was presented. However, the time-frequency lattice of that transform [39, Fig. 1] deviates somewhat from the wavelet time-frequency sampling schema. (In that transform, every second scale is sampled at the same rate as the adjacent scale.) On the other hand, the overcomplete rational DWT with dilation factor $3 / 2$, developed below, is also three-times overcomplete, but it has a more desirable time-frequency lattice than 
the higher-density DWT of [39].

\section{Polynomial Signal Properties of the Rational Filter Bank}

In this paper, for the construction of perfect reconstruction rational filter banks for the implementation of the rational discrete wavelet transform, we will ask of the filters $H(z)$ and $G(z)$ that they satisfy two conditions. For a fixed $N$ and $K$ :

1) We ask that the high-pass analysis channel annihilate discrete-time polynomials of degree $K-1$.

2) We ask that the low-pass analysis and synthesis channels of the filter bank preserve the set of discrete-time polynomial signals of degree $N-1$.

The motivation for these properties is the sparse wavelet representation of piece-wise smooth signals and the smoothness of the analysis/synthesis functions (discrete-time wavelets) [45].

We ask that the filter bank satisfy the first property so that smooth ('locally polynomial') segments of the input signal produce wavelet coefficients that are small in absolute value. In order that this polynomial annihilation property of the high-pass channel be effective for subsequent stages of an iterated filter bank, it is also necessary that the low-pass analysis channel preserve the set of polynomial signals of at least degree $K-1$. Therefore, in the second property we ask that the low-pass analysis channel preserve the set of polynomial signals, and we should have $N \geq K$. In the second property, we might use $N>K$ so as to make the associated discrete-time wavelets more smooth. The same method (using $N$ strictly greater than $K$ ) is also used in the design of dyadic wavelet frames [13], [37] so as to obtain smoother wavelets. Note that in an orthonormal critically-sampled filter bank $N$ and $K$ will be equal. In this paper, however, we will develop overcomplete transforms (frames) so we may have $N>K$.

In the second property we ask also that the low-pass synthesis channel preserve the set of polynomial signals of degree $N-1$. That is because, in the reconstruction of a signal from its wavelet representation, the wavelet coefficients in some scales may be (nearly) set to zero for the purpose of noise reduction or compression, and in such cases it is desirable that the reconstructed signal be smooth and free of unnecessary discontinuities, etc. Additionally, we can view both the analysis and synthesis low-pass channels as fractional rate-changers. As such, we can draw on concepts for the design and analysis of fractional rate changers. A requirement one can ask of a discrete-time rate-changer is that if its input signal is a sampled version of some simple continuous-time signal, then its output signal should correctly resample that continuous-time signal. A basic form of that requirement, is that a discrete-time rate-changer preserve the class of polynomial signals of some degree $N-1$. 


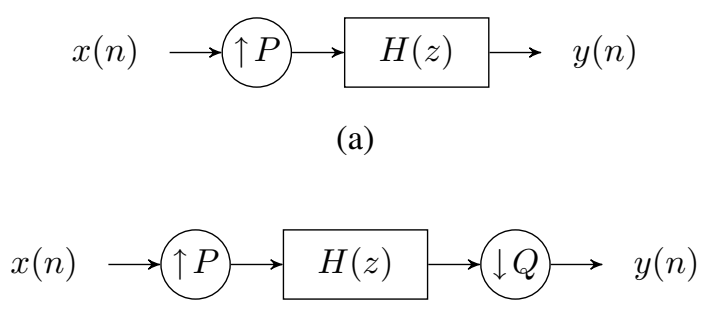

(b)

Fig. 4. (a) Discrete-time $P$-fold interpolator. (b) Fractional $(P / Q)$ rate-changer.

Vanishing moments are an important concept in the construction and understanding of wavelet transforms. A wavelet transform is said to have $K$ vanishing moments if all the wavelet coefficients equal zero whenever the input signal is a polynomial with degree less than $K$. If a filter bank satisfies both properties above, with $N \geq K$, then the discrete wavelet transform has $K$ vanishing moments [14], [38].

The two properties require that $H(z)$ and $G(z)$ satisfy specific conditions. Namely, for the rational filter bank in Fig. 3, the first property requires that $G(z)$ have $\left(1-z^{-1}\right)^{K}$ as a factor, while the second property requires that $H(z)$ have

$$
\left(1+z^{-1}\right)^{N}\left(1+z^{-1}+z^{-2}\right)^{N}
$$

as a factor. To explain the form of this factor, note that the $P$-fold interpolator, illustrated in Fig. 4a, preserves the set of polynomial signals of degree $N-1$ if and only if $H(z)$ has

$$
S_{p}(z):=\left(1+z^{-1}+z^{-2}+\cdots+z^{-(P-1)}\right)^{N}
$$

as a factor [42]. Also, note that down-sampling a discrete-time polynomial signal of degree $N-1$ yields another discrete-time polynomial signal of degree $N-1$. Therefore, the rational rate-changer, illustrated in Fig. 4b, again preserves the set of polynomial signals of degree $N-1$ if and only if $H(z)$ has the term (2) as a factor. Consequently, in order that both the analysis and synthesis low-pass channels of the rational filter bank in Fig. 3 preserve the set of polynomials signals of degree $N-1$, the low-pass filter $H(z)$ must have both $S_{2}(z)$ and $S_{3}(z)$ as factors. Their product yields (1) as a factor.

\section{THE ITERATED LOW-PASS FILTER}

The low-pass branch of the rational analysis filter bank in Fig. 3 is a fractional rate changer. The low-pass subband signal has a lower rate than the input signal (by $2 / 3$ ). In the filter bank implementation 


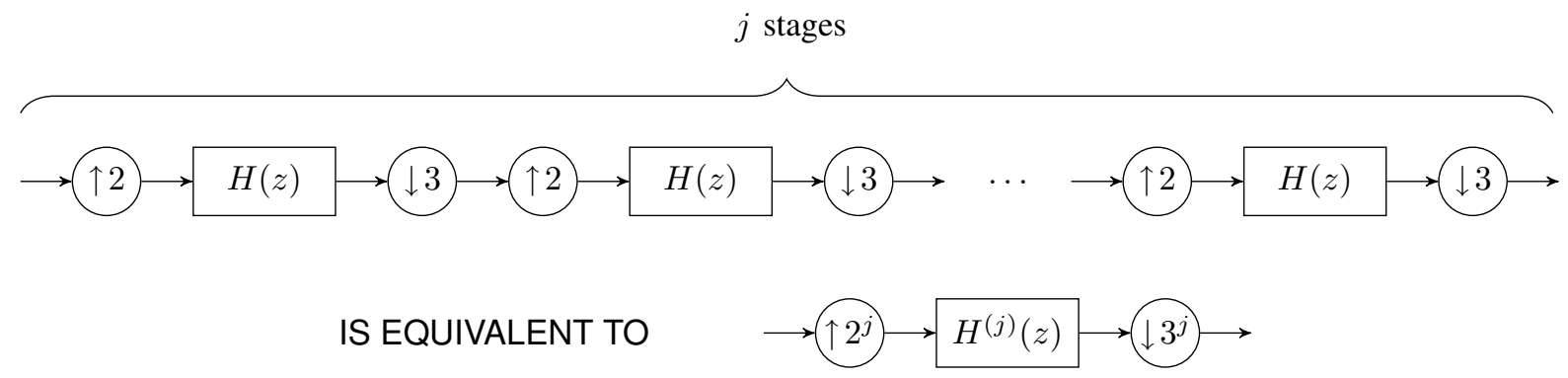

Fig. 5. Iterating a rational rate changer is equivalent to a new rational rate changer. The filter $H^{(j)}(z)$, called the iterated low-pass filter, is given by (3).

of a discrete wavelet transform with rational dilation factors, we iterate a rational filter bank, like the one in Fig. 3, on its low-pass branch. Therefore, the fractional rate changer is connected in series with itself.

When we iterate a 2/3-rate changer $j$ times in succession, we obtain a rate changer that reduces the rate by $(2 / 3)^{j}$. This is equivalent to a single rational rate changer as illustrated in Fig. 5. The equivalent filter $H^{(j)}(z)$, which we call the iterated low-pass filter, is given by

$$
H^{(j)}(z)=\prod_{k=0}^{j-1} H\left(z^{2^{j-1-k} 3^{k}}\right) .
$$

For example, $H^{(3)}(z)=H\left(z^{4}\right) H\left(z^{6}\right) H\left(z^{9}\right)$. Formula (3) is derived using noble identities [44].

For wavelet transforms that are implemented by filter bank iteration, it is desirable to understand the behavior of the filter bank when it is iterated many times. For dyadic wavelet transforms, it is sufficient to check the convergence behaviour of the iterated low-pass filter because it is directly related to the analysis/synthesis functions. We can, in turn, utilize the scaling function $\phi(t)$ because it resembles the iterated low-pass filter $h^{(j)}(n)$ as $j$ goes to infinity. Thus, it is often unnecessary to inspect the iterated low-pass filter of a dyadic filter bank - one can inspect the scaling function $\phi(t)$ instead, to evaluate the limiting behavior of the iterated filter bank. It is generally desirable that $\phi(t)$ be smooth, etc.

However, for a rational WT, there is no simply computed scaling function from which the limiting behaviour of the filter bank can be inferred. Moreover, for the rational WT, the iterated low-pass filter $h^{(j)}(n)$ is not directly related to the analysis/synthesis functions; as described in the next section, it is more appropriate to check the convergence of the polyphase components of $h^{(j)}(n)$ rather than $h^{(j)}(n)$ itself. (Blu and Rioul study the convergence of these polyphase sequences in [7], [36].) Even so, for convenience, in the subsequent examples, we will illustrate the iterated lowpass filter for $j=3$ levels of iteration. 


\section{ORthonormal Rational Discrete WaVelet Transforms}

As mentioned in the introduction, despite the difficulty of constructing a rational wavelet basis on the real line, it is nevertheless straightforward to implement a rational wavelet transform for discrete-time signals. One only needs to iterate the rational filter bank in Fig. 3 on its low-pass branch, where the filters $H(z)$ and $G(z)$ are designed so that the filter bank has the perfect reconstruction property. That constitutes an invertible rational discrete wavelet transform. However, problems remain: the behavior of the filter bank when iterated and the design of perfect reconstruction filters, both of which we discuss here.

First, recall that in a dyadic DWT each wavelet coefficient $w(j, k)$ in a fixed scale, say $j$, of the wavelet representation is the inner product of the input signal $x(n)$ and a translated (and time-reversed) version of the band-pass sequence $g_{\text {dyadic }}^{(j)}(n)$,

$$
w_{\text {dyadic }}(j, k)=\sum_{n} g_{\text {dyadic }}^{(j)}\left(2^{j} k-n\right) x(n),
$$

for $j \geq 1$. The finest scale corresponds to $j=1$. The sequence $g_{\text {dyadic }}^{(j)}(n)$ is the 'discrete-time wavelet' at scale $j$. It is given by basic multirate identities as

$$
G_{\text {dyadic }}^{(j)}(z)=H_{\text {dyadic }}^{(j-1)}(z) G\left(z^{2^{j-1}}\right)
$$

where $H_{\text {dyadic }}^{(j)}(z)$ is the iterated low-pass filter for the dyadic case,

$$
H_{\text {dyadic }}^{(j)}(z)=\prod_{k=0}^{j-1} H\left(z^{2^{k}}\right) .
$$

Note that $H_{\text {dyadic }}^{(0)}(z)=1, G_{\text {dyadic }}^{(1)}(z)=G(z)$, and $H_{\text {dyadic }}^{(1)}(z)=H(z)$. See [45, p. 152].

However, for a rational discrete wavelet transform, the wavelet coefficients in scale $j$ are the inner product of $x(n)$ with translates of several different sequences. That means, there is no unique discrete-time wavelet at a fixed scale $j$. For coarser scales (higher $j$ ), the number of different discrete-time wavelets (basis functions) grows. To clarify, the wavelet coefficients at scale $j$ are given by

$$
w(j, k)=\sum_{n} g^{(j)}\left(3^{j} k-2^{j-1} n\right) x(n)
$$

where

$$
G^{(j)}(z)=H^{(j-1)}(z) G\left(z^{3^{j-1}}\right)
$$

and $H^{(j)}(z)$ is the iterated low-pass filter in (3). As a consequence of (4), at scale $j$, the wavelet coefficients are the inner product of $x(n)$ and the translates of the $2^{j-1}$ polyphase components of $g^{(j)}(n)$. 
For example, at scale $j=2$, there are two discrete-time wavelets: $g^{(2)}(2 n)$ and $g^{(2)}(2 n-1)$. At the finest scale, $j=1$, there is a single discrete-time wavelet, which is simply the high-pass filter impulse response $g(n)$. In the limit as $j$ goes to infinity, there is no uniquely defined wavelet, in contrast to the dyadic case. It is said that the dyadic discrete wavelet transform is time-varying, but the rational discrete wavelet transform is time-varying in this additional sense.

The design of perfect reconstruction filters is also more difficult in the rational case. Recall that in the dyadic case, the design can be accomplished by setting the low-pass filter $H(z)$ equal to a spectral factor of a suitably designed half-band filter [41], [16]. This approach allows one to construct minimal-length perfect reconstruction filters having a specified number of vanishing moments [16]. Unfortunately, this spectral factorization approach can not be used for the design of critically-sampled rational filter banks. Alternative methods for the design of orthonormal rational filter banks have been proposed by Kovačević and Vetterli [29] and Blu [9]. Solutions with more than one vanishing moment were constructed for the first time (to our knowledge) using Gröbner bases [5]. However, even in [5] only a few examples of orthonormal rational filter banks could be constructed: minimal-length orthonormal filters with 1, 2, and 3 vanishing moments and dilation factor $3 / 2$. In principle, Gröbner bases provide a powerful method for solving simultaneous multivariate polynomial equations, but they are limited to small problems in practice due to the long computation time and high memory requirements [32].

Figure 6, which illustrates a minimal-length solution with two vanishing moments from [5], shows the time-varying behavior of the transform. The top panel of Fig. 6 illustrates the frequency response magnitudes $|H(\omega)|$ and $|G(\omega)|$ of the low-pass and high-pass filters. The low-pass filter has double zeros at $\omega=2 \pi / 3$ and at $\omega=\pi$ because $H(z)$ has $\left(1+z^{-1}\right)^{2}\left(1+z^{-1}+z^{-2}\right)^{2}$ as a factor. The middle panel illustrates the iterated low-pass filter $h^{(3)}(n)$. Even though the system has two vanishing moments, the iterated filter is already rough at level 3. As $j$ increases, the iterated low-pass filter $h^{(j)}(n)$ remains extremely rough. The bottom panel of Fig. 6 illustrates two of the 32 discrete-time basis functions (wavelets) at scale $j=6$. In a dyadic DWT, at a single scale, all the discrete-time basis functions are translates of a single discrete-time sequence. However, in the rational case as illustrated in Fig. 6, the basis functions at a single scale can differ from each other substantially.

One way to reduce the shift-variance apparent in the bottom panel of Fig. 6 is to use filters with improved frequency selectivity [9]. We show below that an overcomplete rational FB (provided one can afford the incurred data expansion) can also be used to reduce the shift-variance. 

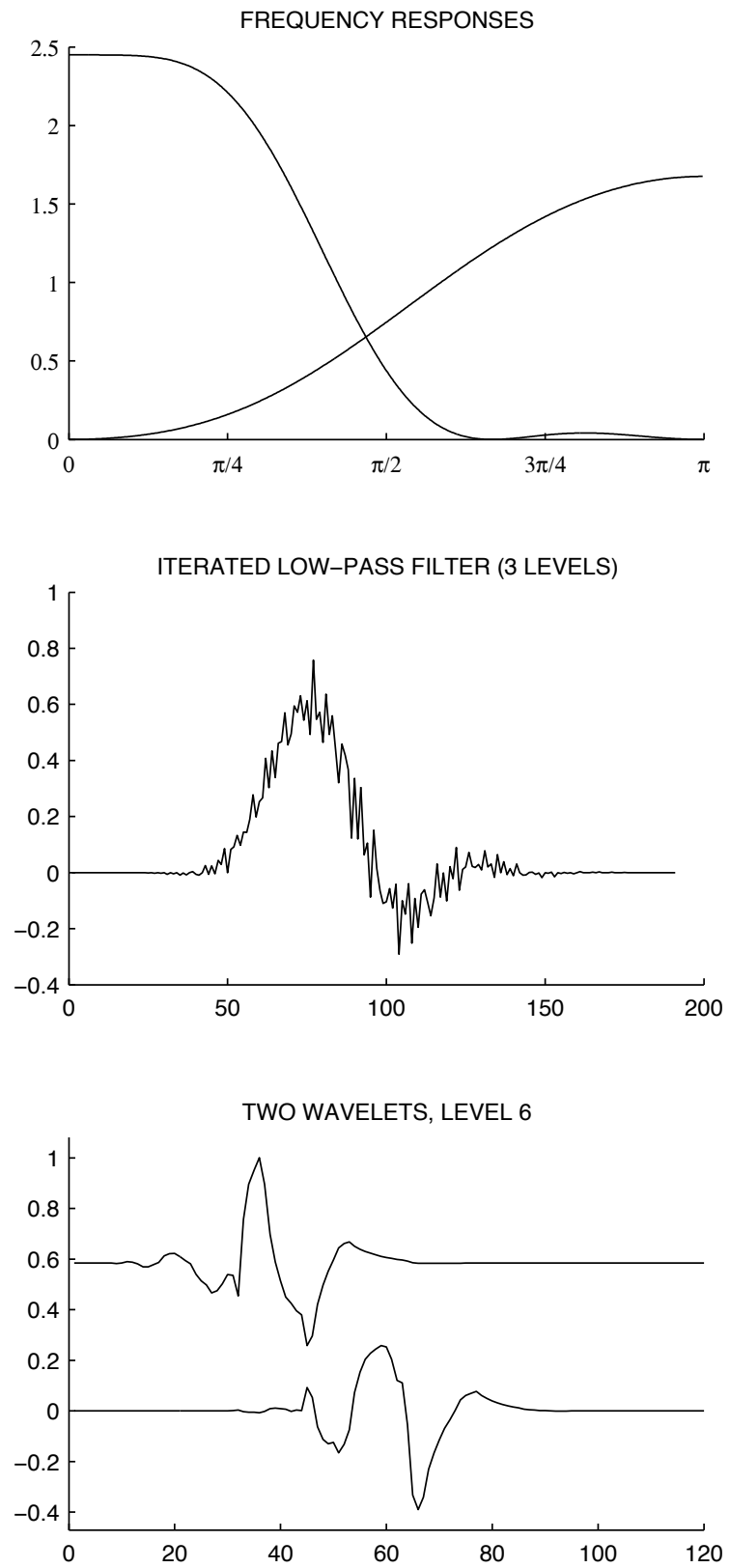

Fig. 6. Example from [5] of minimal-length filters for an orthonormal rational discrete wavelet transform with two vanishing moments and dilation factor 3/2. Top panel: Frequency responses $|H(\omega)|$ and $|G(\omega)|$. Middle panel: The iterated low-pass filter $h^{(3)}(n)$. Bottom panel: Two of the 32 basis functions at scale $j=6$. 


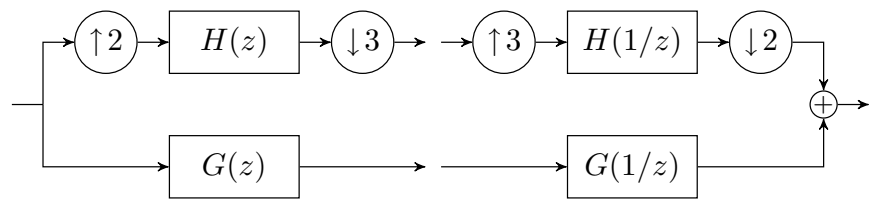

Fig. 7. Partially shift-invariant overcomplete rational filter bank. It attains the time-frequency lattice illustrated in the bottom panel of Fig. 2. However, this filter bank does not allow perfect reconstruction with useful filters which have rational transfer functions.

\section{Partially Shift-Invariant Overcomplete Rational Filter Banks}

As discussed in Section I-A, the sampling of the time-frequency plane illustrated in the bottom panel of Fig. 2 is denser in both time and frequency than that of the critically-sampled dyadic wavelet transform. We are interested in implementing a 'self-inverting' fully discrete transform that corresponds to this oversampled time-frequency lattice. By 'self-inverting' [40], it is meant that the transpose of the transform can be used to invert the transform (the frame is a tight-frame). Moreover, we are interested in developing a discrete transform that can be implemented using iterated filter banks, especially with FIR filters. Note that a PR filter bank is self-inverting when the synthesis filters are the time-reversed versions of the analysis filters.

The desired time-frequency lattice is attained using the over-sampled rational filter bank illustrated in Fig. 7. The high-pass subband has the same rate as the input signal while the low-pass subband has $2 / 3$ that rate. This is a 'partially' shift-invariant filter bank because only the high-pass channel is shift-invariant. Unfortunately, this filter bank can not have the perfect reconstruction property using FIR filters except for a trivial solution. The trivial solution is: $H(z)=0, G(z)=z^{k}$, with $k \in \mathbb{Z}$. A proof is provided in the Appendix.

\section{A. Ideal Stop-bands and Perfect Reconstruction}

Although the partially shift-invariant filter bank precludes useful FIR solutions, effective filters can still be designed by their frequency responses and an exactly PR system can be implemented using the FFT. To develop this type of solution we need the PR conditions in the frequency domain:

$$
\begin{array}{r}
|H(\omega)|^{2}+|H(\omega-\pi)|^{2}+6|G(2 \omega)|^{2}=6, \\
H(\omega+2 \pi / 3) H(-\omega)+H(\omega-\pi / 3) H(\pi-\omega)=0, \\
H(\omega-2 \pi / 3) H(-\omega)+H(\omega+\pi / 3) H(\pi-\omega)=0 .
\end{array}
$$


Note that if the low-pass filter has an ideal stop-band with stop-band edge $\pi / 3$, that is if $H(\omega)=0$ for all $\pi / 3 \leq|\omega| \leq \pi$, then (5b) and (5c) are satisfied. The frequency response $H(\omega)$ for $|\omega| \leq \pi / 3$ can then be chosen freely (i.e. $H(\omega)$ need not be an ideal filter), subject to $|H(\omega)| \leq \sqrt{6}$. Then $G(\omega)$ is defined from (5a) and depends on the chosen function $H(\omega)$. In this paper we are interested in FIR filter banks; therefore we do not further develop this direction.

\section{OVERCOMPLETE FIR RATIONAL FILTER BANKS}

We must forgo the partially shift-invariant filter bank illustrated in Fig. 7 because we are interested in FIR perfect reconstruction solutions. As an alternative, we will develop solutions for the filter bank illustrated in Fig. 8. Note that if the filters $G_{i}(z)$ in Fig. 8 are translates of each other,

$$
g_{2}(n)=g_{1}(n-1)=g_{0}(n-2),
$$

then the filter bank in Fig. 8 is essentially the same as the one in Fig. 7 and therefore it does not admit useful FIR PR solutions. (Interlacing the $G_{i}$ subbands in Fig. 8 produces the $G$ subband in Fig. 7.) However the two filter banks are generally not equivalent, the one in Fig. 8 being less constrained.

In this section we will obtain FIR PR solutions for Fig. 8 which satisfy (6) approximately, and which also satisfy the polynomial properties described in Section II. We will first design the low-pass filter $H(z)$ which must satisfy specific conditions to be described. Second, given $H(z)$ we will find $G_{i}(z)$ to 'complete' the filter bank (so that the filter bank has the perfect reconstruction property).

The PR conditions for the filter bank in Fig. 8 are given by:

$$
\begin{gathered}
H(z) H(1 / z)+H(-z) H(-1 / z)+2 \sum_{i=0}^{2} G_{i}\left(z^{2}\right) G_{i}\left(1 / z^{2}\right)=6, \\
H(W z) H(1 / z)+H(-W z) H(-1 / z)+2 \sum_{i=0}^{2} G_{i}\left(W^{2} z^{2}\right) G_{i}\left(1 / z^{2}\right)=0, \\
H\left(W^{2} z\right) H(1 / z)+H\left(-W^{2} z\right) H(-1 / z)+2 \sum_{i=0}^{2} G_{i}\left(W z^{2}\right) G_{i}\left(1 / z^{2}\right)=0 .
\end{gathered}
$$

For the design and analysis of the FB in Fig. 8, it will be useful below to consider also the equivalent system shown in Fig. 9, where the filters $H_{0}(z)$ and $H_{1}(z)$ are the polyphase components of $H(z)$ defined by

$$
H(z)=H_{0}\left(z^{2}\right)+z^{-3} H_{1}\left(z^{2}\right) .
$$

It is more common to use $z^{-1}$ for defining the polyphase component $H_{1}(z)$, however, the use of $z^{-3}$ will simplify numerous subsequent expressions. 


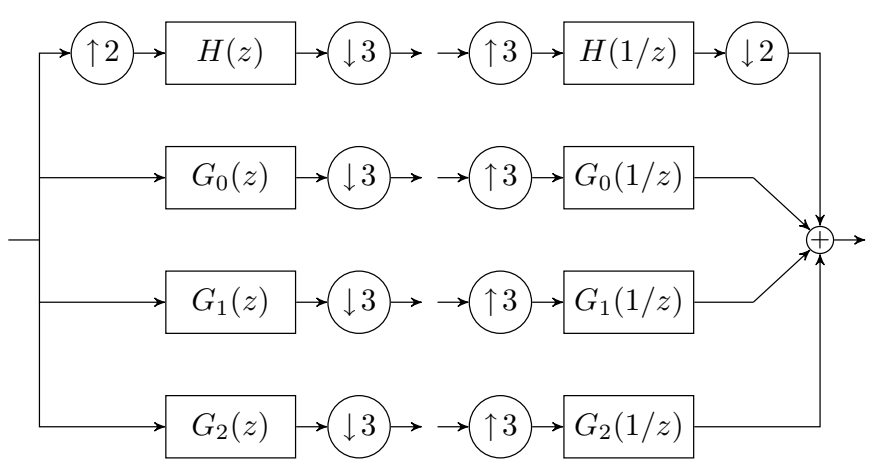

Fig. 8. An overcomplete rational filter bank. This filter bank is less constrained than the one in Fig. 7 and useful FIR perfect reconstruction filters for it can be designed.

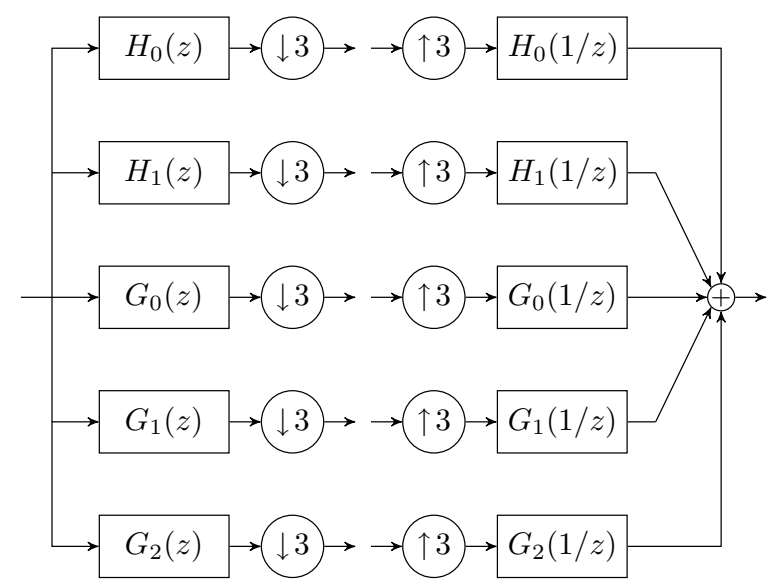

Fig. 9. A filter bank that is equivalent to the one in Fig. 8.

\section{A. Designing the Low-Pass Filter $H(z)$}

We would like to construct an FIR low-pass filter $H(z)$ so that the overcomplete rational filter bank in Fig. 8 can be completed. As discussed in Section II, given $N$ and $K$ (with $N \geq K$ ), we would like $H(z)$ to be of the form

$$
H(z)=\left(1+z^{-1}\right)^{N}\left(1+z^{-1}+z^{-2}\right)^{N} Q(z)
$$

and we would like to ensure, once the filter bank is completed, that the three FIR high-pass filters $G_{i}(z)$ will be of the form

$$
G_{i}(z)=\left(1-z^{-1}\right)^{K} V_{i}(z), \quad i=0,1,2 .
$$


Using (9) and (10) in (7) gives

$$
\begin{array}{r}
H(z) H(1 / z)+\left(-z+2-z^{-1}\right)^{K}\left[\left(-z+2-z^{-1}\right)^{N-K}\left(-z+1-z^{-1}\right)^{2 N} Q(-z) Q(-1 / z)\right. \\
\left.+2\left(z+2+z^{-1}\right)^{K} \sum_{i=0}^{2} V_{i}\left(1 / z^{2}\right) V_{i}\left(z^{2}\right)\right]=6 .
\end{array}
$$

Therefore, it is required from (9) and (11) that the product filter,

$$
P(z):=H(z) H(1 / z)
$$

simultaneously have the two forms

$$
\begin{aligned}
& P(z)=\left(z+2+z^{-1}\right)^{N}\left(z+1+z^{-1}\right)^{2 N} R_{1}(z) \\
& P(z)=6-\left(-z+2-z^{-1}\right)^{K} R_{2}(z)
\end{aligned}
$$

for some Type-I FIR filters $R_{1}(z)$ and $R_{2}(z)$. The condition (13) puts nulls in the stop-band of the frequency response of $P(z)$. The condition (14) makes the frequency response of $P(z)$ flat at dc (i.e. several derivatives of the frequency response $P\left(e^{j \omega}\right)$ are zero at $\left.\omega=0\right)$.

For given $N$ and $K$, to obtain a low-pass filter $H(z)$ of minimum degree, we will first construct the minimum degree $P(z)$ satisfying (13) and (14). Then we will obtain $H(z)$ from $P(z)$ by spectral factorization (provided $P(z)$ admits spectral factorization). Note that $H(z)$ will not be uniquely determined from $P(z)$.

To obtain $P(z)$, we utilize the transformation,

$$
x=\frac{1}{4}\left(1-z^{-1}\right)(1-z),
$$

and write

$$
P(z)=F(x)
$$

for some polynomial $F(x)$. This transformation can always be used to transform a Type-I filter into a polynomial. We will first obtain $F(x)$ and then get $P(z)$ by substitution of (15). First, note that

$$
\begin{aligned}
(1-x) & =\frac{1}{4}\left(z+2+z^{-1}\right) \\
(3 / 4-x) & =\frac{1}{4}\left(z+1+z^{-1}\right) .
\end{aligned}
$$

Then using (17) and (18) in (13) and (14) gives the two conditions

$$
\begin{aligned}
& F(x)=(1-x)^{N}(3 / 4-x)^{2 N} A(x) \\
& F(x)=6+x^{K} B(x)
\end{aligned}
$$


for some polynomials $A(x)$ and $B(x)$. Combining (19) and (20) gives the equation,

$$
(1-x)^{N}(3 / 4-x)^{2 N} A(x)-x^{K} B(x)=6,
$$

or

$$
A(x)=\left[(1-x)^{N}(3 / 4-x)^{2 N}\right]^{-1}\left(6+x^{K} B(x)\right) .
$$

Now if we consider the following power series around $x=0$,

$$
\begin{gathered}
\frac{1}{(1-x)^{N}}=\sum_{n=0}^{\infty} a_{n} x^{n}, \\
\frac{1}{(3 / 4-x)^{N}}=\sum_{n=0}^{\infty} b_{n} x^{n}
\end{gathered}
$$

with

$$
\begin{aligned}
a_{n} & =\left(\begin{array}{c}
n+N-1 \\
N-1
\end{array}\right) \\
b_{n} & =\left(\frac{4}{3}\right)^{N}\left(\begin{array}{c}
n+N-1 \\
N-1
\end{array}\right)\left(\frac{4}{3}\right)^{n},
\end{aligned}
$$

we have, from (22),

$$
A(x)=\left(\sum_{n=0}^{\infty} c_{n} x^{n}\right)\left(6+x^{K} B(x)\right)
$$

where

$$
c_{n}=a_{n} * b_{n} * b_{n}
$$

From the Euclidian algorithm for polynomial GCDs, we see that $A(x)$ and $B(x)$ are of degree $K-1$ and $3 N-1$ respectively, therefore

$$
A(x)=6 \sum_{n=0}^{K-1} c_{n} x^{n}
$$

We can thus obtain the polynomial $F(x)$ in (19), and in turn the filter $P(z)$ in (16), which in turn gives $H(z)$ by spectral factorization from (12).

Example 1: For $N=3, K=1$, we get the low-pass filter

$$
H(z)=\frac{\sqrt{6}}{6^{3}}\left(1+z^{-1}\right)^{3}\left(1+z^{-1}+z^{-2}\right)^{3} .
$$

Example 2: For $N=4, K=2$, we get the low-pass filter

$$
H(z)=\frac{\sqrt{6}}{3+\sqrt{141}}\left(25+\sqrt{141}-22 z^{-1}\right)\left(\frac{1+z^{-1}}{2}\right)^{4}\left(\frac{1+z^{-1}+z^{-2}}{3}\right)^{4} .
$$

Example 3: For $N=5, K=3$, we get the low-pass filter

$$
H(z)=\left(q_{0}+q_{1} z^{-1}+q_{2} z^{-2}\right)\left(\frac{1+z^{-1}}{2}\right)^{5}\left(\frac{1+z^{-1}+z^{-2}}{3}\right)^{5} .
$$

with $q_{0}=13.51216939, q_{1}=-16.04275832, q_{2}=4.98007867$. 


\section{B. Completing the Filter Bank}

Given the low-pass filter $H(z)$, in this section we show how to complete the filter bank in Fig. 8 so that the filter bank has the perfect reconstruction property. Equivalently, given $H_{0}(z)$ and $H_{1}(z)$, the problem is to find $G_{i}(z)$ so that the filter bank in Fig. 9 has the PR property. To this end, we define the polyphase components of $H_{0}(z)$ through

$$
H_{0}(z)=H_{00}\left(z^{3}\right)+z^{-1} H_{01}\left(z^{3}\right)+z^{-2} H_{02}\left(z^{3}\right) .
$$

The polyphase components of $H_{1}(z)$ and $G_{i}(z)$ are similarly defined. By standard manipulations, the filter bank is PR if and only if the associated polyphase matrix satisfies

$$
\left[\begin{array}{ll}
\mathbf{H}^{T}(1 / z) & \mathbf{G}^{T}(1 / z)
\end{array}\right]\left[\begin{array}{l}
\mathbf{H}(z) \\
\mathbf{G}(z)
\end{array}\right]=\mathbf{I}_{3}
$$

where

$$
\begin{aligned}
& \mathbf{H}(z):=\left[\begin{array}{lll}
H_{00}(z) & H_{01}(z) & H_{02}(z) \\
H_{10}(z) & H_{11}(z) & H_{12}(z)
\end{array}\right], \\
& \mathbf{G}(z):=\left[\begin{array}{lll}
G_{00}(z) & G_{01}(z) & G_{02}(z) \\
G_{10}(z) & G_{11}(z) & G_{12}(z) \\
G_{20}(z) & G_{21}(z) & G_{22}(z)
\end{array}\right] .
\end{aligned}
$$

From (34) we have

$$
\mathbf{G}^{T}(1 / z) \mathbf{G}(z)=\mathbf{I}_{3}-\mathbf{H}^{T}(1 / z) \mathbf{H}(z) .
$$

Therefore we can obtain $\mathbf{G}(z)$ by performing matrix spectral factorization on the matrix $\mathbf{F}(z):=\mathbf{I}_{3}-$ $\mathbf{H}^{T}(1 / z) \mathbf{H}(z)$ which depends on the low-pass filter $H(z)$ only. This requires that the matrix $\mathbf{F}(z)$ be positive-definite for $z$ on the unit circle. (We note, for the low-pass filter developed in Section VI-A, it can be shown that the determinant of the matrix $\mathbf{F}(z)$ contains $(1-z)^{K}\left(1-z^{-1}\right)^{K}$ as a factor. Therefore positive definiteness of $\mathbf{F}(z)$ will be sought on the unit circle except for a finite number of points.)

\section{Algorithms for Matrix Spectral Factorization}

Most of the computational work of the rational filter bank design method formulated in this paper is the matrix spectral factorization problem: Given $\mathbf{F}(z)$, find $\mathbf{G}(z)$ such that

$$
\mathbf{G}^{T}(1 / z) \mathbf{G}(z)=\mathbf{F}(z)
$$

This factorization is also called the matrix-valued Fejér-Riesz lemma [23]. 
A variety of algorithms have been developed to solve the matrix spectral factorization problem, as early as [46] and as recently as [21]. An overview of some algorithms are given in [31]. Some methods are iterative and avoid explicitly computing roots of polynomials — but these methods often yield only minimum-phase solutions. We wish to compute multiple factorizations, for example so that we may select a factorization that generates 'least asymmetric' wavelets. Therefore, we use the symmetric factor extraction algorithm [30], [31].

The symmetric factor extraction algorithm begins by performing scalar spectral factorization of the determinant of $\mathbf{F}(z)$. (For the matrix $\mathbf{F}(z)$ arising in this paper, the zero of $\operatorname{det} \mathbf{F}(z)$ at $z=1$ of multiplicity $2 K$ must be properly taken into account so as to avoid the loss of numerical accuracy.) Then the degree is gradually reduced using carefully selected simple matrices, until a unimodular matrix is obtained. Next, this unimodular matrix is factored using another type of factor, yielding a positive-definite symmetric constant matrix, which is then itself factored. This type of algorithm was developed initially for continuous-time systems [17], [11] and later updated and modified for the discrete-time case [34].

In signal processing, matrix spectral factorization is well-known for multichannel spectral estimation and optimal filtering [35]. It has been used for the design of wavelet bases, frames, and filter banks in [14], [23], [15]. The papers [14], [23] also describe spectral factorization algorithms (for the $2 \times 2$ case in [14]) which use scalar spectral factorization and linear algebra. Also [22] considers the $2 \times 2$ case where each factor is itself symmetric.

\section{Necessary and Sufficient Conditions for Completion}

Given a low-pass filter $H(z)$, how can we quickly check if the overcomplete filter bank in Fig. 8 can be completed so as to be PR? We must check that $\mathbf{I}_{3}-\mathbf{H}^{T}(1 / z) \mathbf{H}(z)$ is positive-definite (on all $|z|=1$ except a finite number of points.). It can be shown that this is equivalent to checking that the smaller matrix, $\mathbf{I}_{2}-\mathbf{H}(z) \mathbf{H}^{T}(1 / z)$, is positive-definite. This is expected since 1 is always an eigenvalue of $\mathbf{I}_{3}-\mathbf{H}^{T}(1 / z) \mathbf{H}(z)$. To check that a matrix is positive-definite, we can check the positive definiteness of all the upper left submatrices of the matrix [43]. This gives us the following two conditions:

$$
\begin{gathered}
1-\sum_{k=0}^{2} H_{0 k}(z) H_{0 k}(1 / z)>0 \\
\left(1-\sum_{k=0}^{2} H_{0 k}(z) H_{0 k}(1 / z)\right)\left(1-\sum_{k=0}^{2} H_{1 k}(z) H_{1 k}(1 / z)\right) \\
-\left(\sum_{k=0}^{2} H_{0 k}(z) H_{1 k}(1 / z)\right)\left(\sum_{k=0}^{2} H_{1 k}(z) H_{0 k}(1 / z)\right)>0 .
\end{gathered}
$$


for $|z|=1$. Using the AC matrix [44] ${ }^{1}$ of $\mathbf{H}(z)$ we obtain equivalent conditions,

$$
\begin{gathered}
3-\sum_{k=0}^{2}\left|H_{i}(\omega+2 k \pi / 3)\right|^{2}>0, \\
\left(3-\sum_{k=0}^{2}\left|H_{0}(\omega+2 k \pi / 3)\right|^{2}\right)\left(3-\sum_{k=0}^{2}\left|H_{1}(\omega+2 k \pi / 3)\right|^{2}\right) \\
-\left|\sum_{k=0}^{2} H_{0}(\omega+2 k \pi / 3) H_{1}^{*}(\omega+2 k \pi / 3)\right|^{2}>0 .
\end{gathered}
$$

Notice that the functions above are periodic with period $2 \pi / 3$, so it suffices to check these on $\omega \in$ $[0,2 \pi / 3]$. Note that if the filters $H_{i}(z)$ were part of an orthonormal $\mathrm{FB}$, then these inequalities hold with equality.

Example: To check that the low-pass filters (30), (31), and (32) can be completed to a tight frame, we evaluate (40) and (41) using the polyphase components (8). The functions (not shown to conserve space) are nonnegative; therefore the filter bank can be completed to have the perfect reconstruction property for each of the three low-pass filters considered.

\section{E. Non-uniqueness of Matrix Spectral Factorization}

Notice that the solution to the matrix spectral factorization problem $\mathbf{G}(z)$ is not unique. In particular we have,

$$
\mathbf{G}^{T}(1 / z) \mathbf{G}(z)=\mathbf{G}^{T}(1 / z) \mathbf{R}^{T}(1 / z) \mathbf{R}(z) \mathbf{G}(z)
$$

where $\mathbf{R}(z)$ is any paraunitary matrix. Such paraunitary factors can be introduced so as to modify the frequency responses of $G_{i}(z)$, for example.

\section{F. Approximate Partial Shift-Invariance}

It was shown in Section $\mathrm{V}$ that the overcomplete rational filter bank in Fig. 8 does not admit useful FIR perfect reconstruction filters satisfying the partial shift-invariance property (6). However, we can obtain solutions that satisfy this property approximately,

$$
g_{2}(n) \approx g_{1}(n-1) \approx g_{0}(n-2)
$$

\footnotetext{
${ }^{1}$ The resulting matrices (obtained using the AC matrix and the polyphase matrix) are related by a similarity transformation on the unit circle, so their eigenvalues are the same. Positivity of one of the matrices implies that of the other.
} 
With such solutions, the rational DWT will be (a) approximately shift-invariant, and (b) will be characterized by the time-frequency lattice in the bottom panel of Fig. 2.

In order to obtain solutions satisfying (43) it is required first, that the low-pass filter $H(z)$ be chosen correctly and second, that an appropriate paraunitary matrix $\mathbf{R}(z)$ in (42) be utilized as necessary. The conditions on the low-pass filter can be found by noting that if the filter bank in Fig. 8 is PR and satisfies (43), then $H(z)$ should approximately satisfy the PR conditions (5b), (5c) of the partially shift-invariant filter bank in Fig. 7. Expressed in terms of the two polyphase components $H_{i}(z)$ of $H(z)$ (defined through (8)) these conditions become

$$
H_{0}(\omega \pm 2 \pi / 3) H_{0}(-\omega)+H_{1}(\omega \pm 2 \pi / 3) H_{1}(-\omega) \approx 0 .
$$

Note that $H_{i}(z)$ satisfy (44) if

$$
H_{i}(\omega \pm 2 \pi / 3) H_{i}(-\omega) \approx 0, \quad i=0,1 .
$$

This suggests, for example, that $H_{i}(z)$ are low-pass filters with stop-band edges at $\pi / 3$, namely that $H_{i}(\omega) \approx 0$ for $\pi / 3 \leq|\omega| \leq \pi$. In this case, the condition (44) will be satisfied which implies that the filter bank admits an approximately shift-invariant completion. However (45) is too restrictive and (44) can be approximately satisfied even when (45) is not (see Fig. 10).

This discussion only points out the existence of an approximately shift-invariant set of high-pass filters $G_{i}(z)$ to complete the filter bank given a low-pass filter satisfying (44). It does not suggest how to construct the filters $G_{i}(z)$ given the low-pass filter $H(z)$. The filters $G_{i}(z)$ produced by the matrix spectral factorization procedure will generally be far from satisfying (43). However, they can be manipulated using PR preserving filter bank operations to obtain an approximately shift-invariant solution. A detailed example of that procedure, as applied for the double-density DWT, is described in [38]. The manipulations illustrated in [38] are equivalent to building a paraunitary matrix $\mathbf{R}(z)$ in (42).

Example: Consider the low-pass filter (30). It is known already from Section VI-D that, given this lowpass filter, the rational overcomplete filter bank can be completed so as to have the perfect reconstruction property. Let us now examine if the completion can be approximately shift-invariant (meaning that $g_{i}(n)$ satisfy (43)). To this end, the left hand side of (44) is evaluated and illustrated in the top panel of Fig. 10. Because the function is relatively close to zero, it can be concluded that an approximately shift-invariant filter bank completion is possible. Furthermore, in the lower panel of Fig. 10 the product $\left|H_{0}(\omega-2 \pi / 3) H_{0}(-\omega)\right|$ is illustrated; it is not close to zero - this implies that (45) is too restrictive a condition to check. Similarly, the low-pass filters (31) and (32) also satisfy (44). 

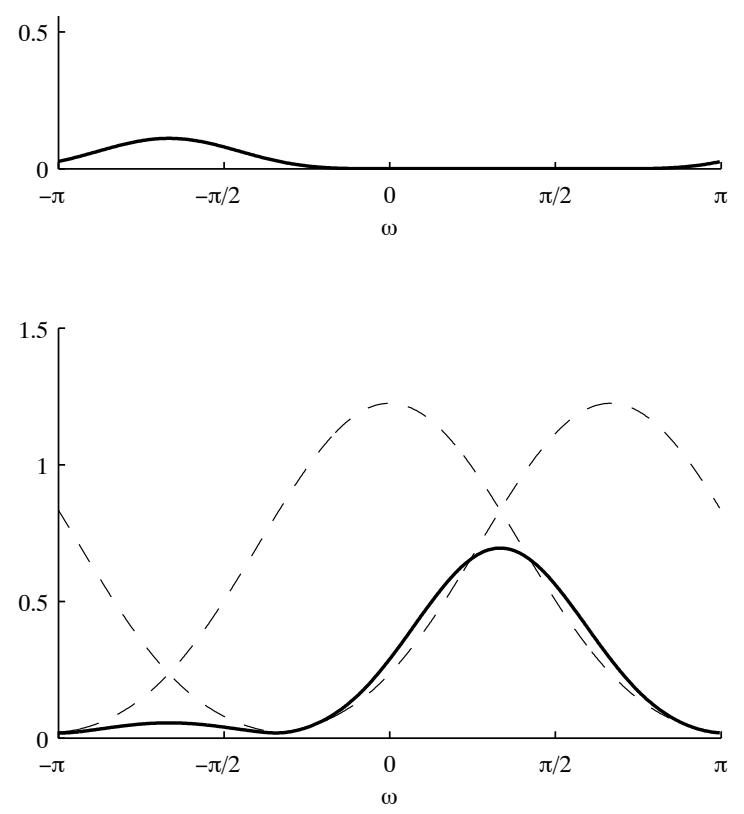

Fig. 10. Top panel: The left-hand-side of (44) for the low-pass filter $H(z)$ in (30). Bottom panel: $\left|H_{0}(\omega-2 \pi / 3)\right|,\left|H_{0}(-\omega)\right|$ and their product (solid). The figure illustrates that $H(z)$ approximately satisfies (44) but not (45), and that (45) is therefore too restrictive.

\section{EXAMPLES}

Example 1: In this example we construct an overcomplete rational DWT (actually a PR rational filter bank, Fig. 8) based on the low-pass filter in (30). The frequency response $|H(\omega)|$ of the low-pass filter is illustrated in the top panel of Fig. 11. The iterated low-pass filter $h^{(3)}(n)$ is illustrated in the middle panel of Fig. 11. Note that the iterated low-pass filter is much smoother than the one arising in the orthonormal case, illustrated in Fig. 6.

We already know that, given the low-pass filter (30), the filter bank admits an approximately shiftinvariant completion. To carry out the completion, we form the matrix $\mathbf{H}(z)$ in (35) using the polyphase components as defined in (8) and (33). Applying the symmetric factor extraction algorithm [30] to perform matrix spectral factorization of $\mathbf{I}_{3}-\mathbf{H}^{T}(1 / z) \mathbf{H}(z)$ in (37), we obtain $\mathbf{G}(z)$. From $\mathbf{G}(z)$ we obtain three filters $G_{i}(z), i=0,1,2$. However, the filters $G_{i}(z)$ are far away from satisfying (43). We next find a paraunitary matrix $\mathbf{R}(z)$ in (42), following the method described in [38], to obtain a new set of filters $G_{i}(z)$ satisfying the approximate shift-invariant property (43). The coefficients of the filters $g_{i}(n)$ are tabulated in Table I.

As described in Section IV, at level $j$ of the rational DWT (where $j=1$ is the finest scale), there 
TABLE I

EXAMPLE 1 - THE HIGH-PASS FILTERS $g_{i}(n)$ COMPLETING THE LOW-PASS FILTER (30) TO AN OVERCOMPLETE RATIONAL FILTER BANK. THE SOLUTION IS ILLUSTRATED IN FIG. 11.

\begin{tabular}{rrrr}
\hline$n$ & $g_{0}(n)$ & $g_{1}(n)$ & $g_{2}(n)$ \\
\hline 0 & 0.64917778505741 & 0 & 0 \\
1 & -0.48262654366226 & 0.63770868747435 & 0 \\
2 & -0.15059130119969 & -0.46687803212812 & 0.64520631583316 \\
3 & -0.01477135217528 & -0.14815175304968 & -0.49098922627425 \\
4 & -0.00118858802016 & -0.02267890229656 & -0.13149490989732 \\
5 & 0 & 0 & -0.02152627546889 \\
6 & 0 & 0 & -0.00119590419272 \\
\hline
\end{tabular}

are $2^{j-1}$ discrete-time analysis/synthesis functions ('wavelets') for each high-pass filter $g_{i}(n)$. Therefore, for the overcomplete rational DWT, there are 96 discrete-time wavelets at level $j=6$. Two of them are illustrated in the bottom panel of Fig. 11. (They are offset vertically for visibility.) In contrast to the orthonormal case illustrated in Fig. 6, the wavelets are very similar to each other and are also substantially smoother.

Note that, by design of the low-pass filter, the overcomplete rational DWT implemented using these filters has only one vanishing moment $(K=1$ in (30)).

Example 2: In this example we construct an overcomplete rational DWT with two vanishing moments by using the low-pass filter in (31). Because this low-pass filter was designed using $K=2$ in Section VI-A, it is ensured that once the filter bank is completed, each of the high-pass filters will have $\left(1-z^{-1}\right)^{2}$ as a factor.

The frequency response $|H(\omega)|$ and the iterated low-pass filter are illustrated in Fig. 12. Again, the iterated low-pass filter is much smoother than that of the orthonormal case. As in Example 1, we obtain an approximately shift-invariant completion of the filter bank. The coefficients of the filters $g_{i}(n)$ are tabulated in Table II.

Recall that part of the matrix spectral factorization algorithm requires the scalar spectral factorization of the determinant of the matrix $\mathbf{F}(z)$. This step has numerous solutions; therefore, numerous filter bank completions are available. Only one example is provided here, namely the one that generates the least asymmetric analysis/synthesis functions (wavelets). Two of the 96 discrete-time wavelets at level $j=6$ 

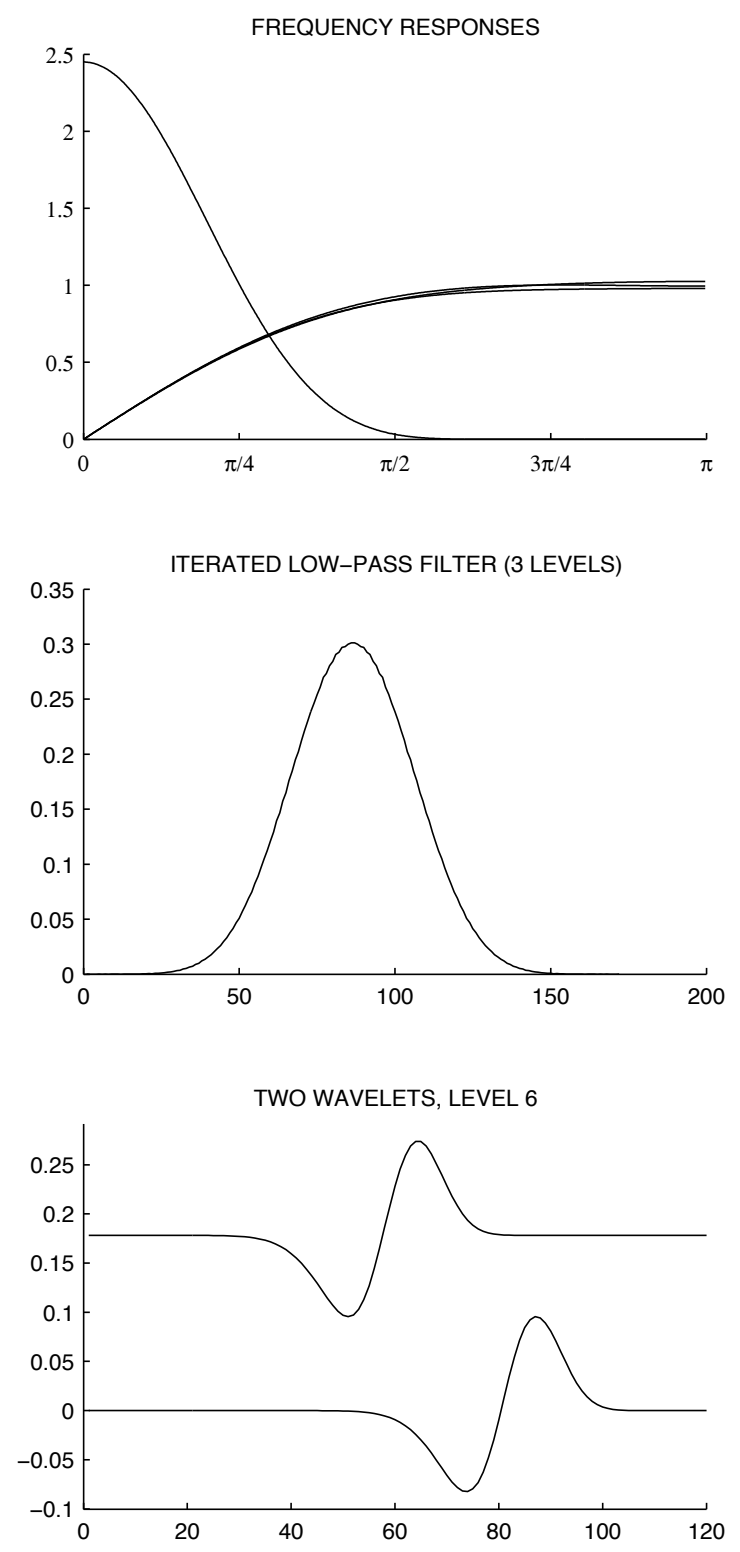

Fig. 11. Example 1 - Top: the low-pass filter in (30) and the high-pass filters in Table I. Middle: the iterated low-pass filter $h^{(3)}(n)$. Bottom: two of the numerous discrete-time analysis/synthesis functions at level $j=6$. Compare to Fig. 6 .

are illustrated in the bottom panel of Fig. 12. For each level $j \geq 1$, all the wavelets are very similar to each other and smooth, in contrast to the orthonormal solution, illustrated in Fig. 6.

It is interesting to note that the wavelets are very nearly symmetric even though the low-pass filter impulse response $h(n)$ is not, nor are the high-pass filters $g_{i}(n)$. Furthermore, the wavelets closely resemble the 'Mexican hat' wavelet. The Mexican hat wavelet is the second derivative of the Gaussian 

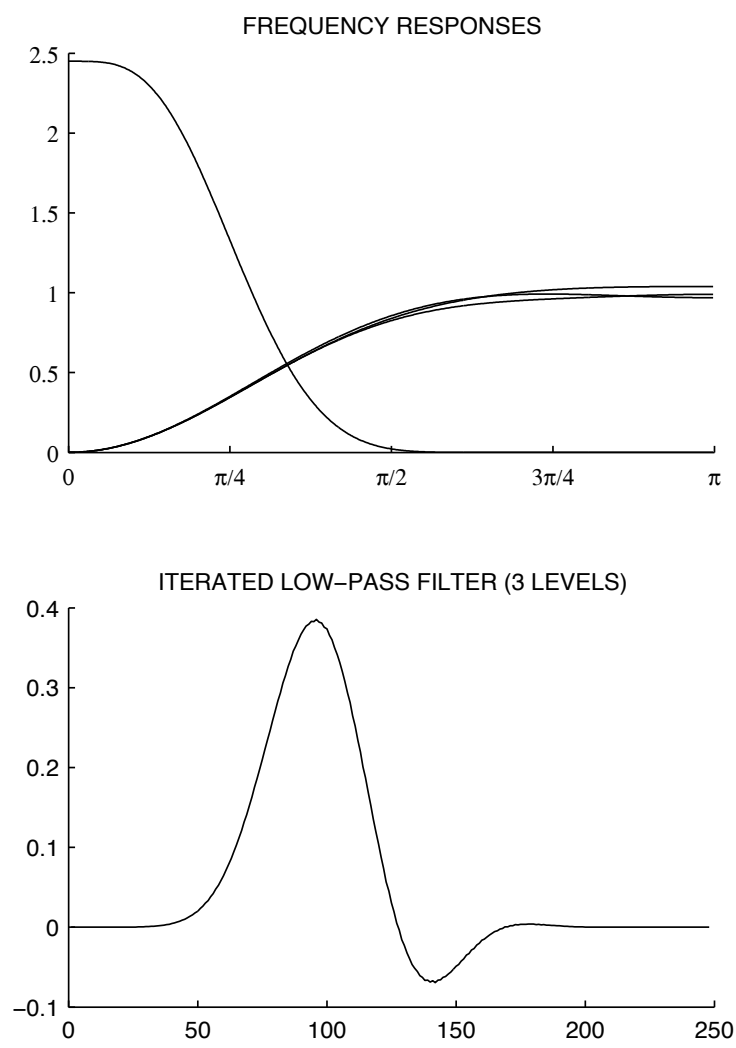

TWO WAVELETS, LEVEL 6

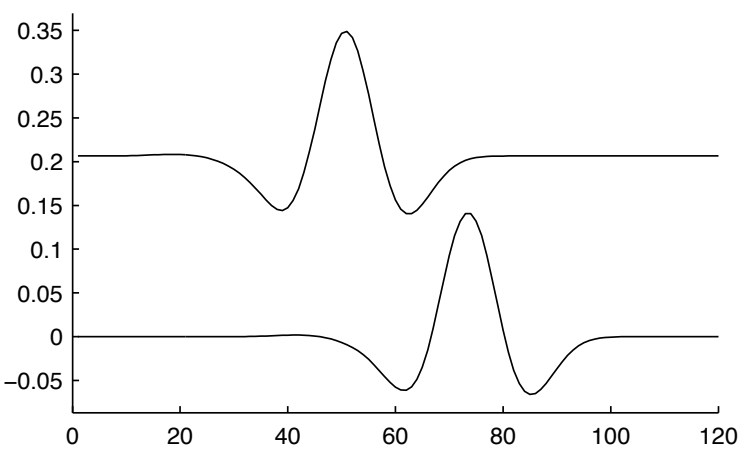

Fig. 12. Example 2 - Top: the low-pass filter in (31) and the high-pass filters in Table II. Middle: the iterated low-pass filter $h^{(3)}(n)$. Bottom: two of the numerous discrete-time analysis/synthesis functions at level $j=6$. Compare to Fig. 6 and Fig. 11 . 
TABLE II

EXAMPLE 2 - THE HIGH-PASS FILTERS $g_{i}(n)$ COMPLETING THE LOW-PASS FILTER (31) TO AN OVERCOMPLETE RATIONAL FILTER BANK. THE SOLUTION IS ILLUSTRATED IN FIG. 12.

\begin{tabular}{rrrr}
\hline$n$ & $g_{0}(n)$ & $g_{1}(n)$ & $g_{2}(n)$ \\
\hline 0 & -0.40908710960769 & 0 & 0 \\
1 & 0.60883534099560 & -0.41859277102914 & 0 \\
2 & -0.04184627465725 & 0.63768171581886 & -0.41711557662580 \\
3 & -0.11027254862108 & -0.06602277318640 & 0.61956405204795 \\
4 & -0.04402437477448 & -0.11231075043309 & -0.03207585686531 \\
5 & -0.00340255359451 & -0.03507318770649 & -0.13086517327386 \\
6 & -0.00020247971016 & -0.00568223335005 & -0.03495288808512 \\
7 & 0 & 0 & -0.00432207685257 \\
8 & 0 & 0 & -0.00023248035976 \\
\hline
\end{tabular}

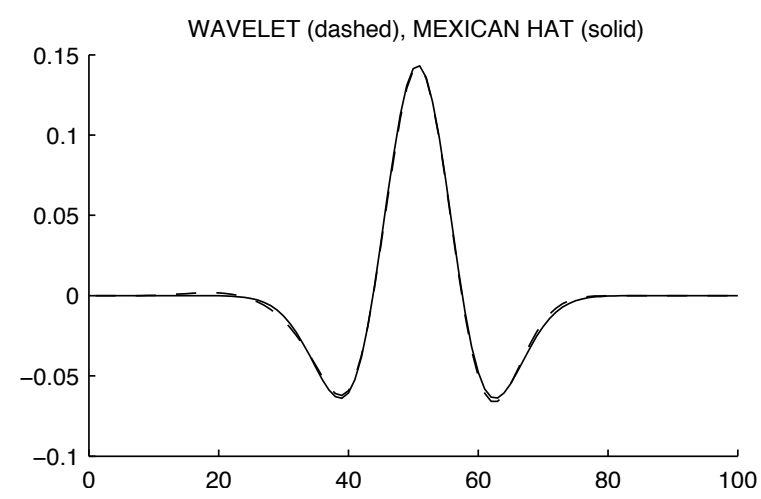

Fig. 13. Example 2 - The discrete-time wavelets are very similar to the Mexican hat function. The plot illustrates one of the discrete-time wavelets (dashed) and the the Mexican hat with parameters chosen to match (solid).

function,

$$
f(t)=A \exp \left(-\frac{(t-\mu)^{2}}{2 \sigma^{2}}\right)
$$

and is often used with the continuous wavelet transform [1], [24] and in scale-space filtering [33]. To illustrate how closely the discrete-time wavelets in this example resemble the Mexican hat function, we find the parameters $A, \mu$, and $\sigma$ in (46) so that $f^{\prime \prime}(t)$ optimally matches the first of the two discrete-time wavelets in the bottom panel of Fig. 12. The resulting function $f^{\prime \prime}(t)$ is illustrated in Fig. 13.

Because the wavelets well approximate the Mexican hat function, and because its time-frequency lattice 


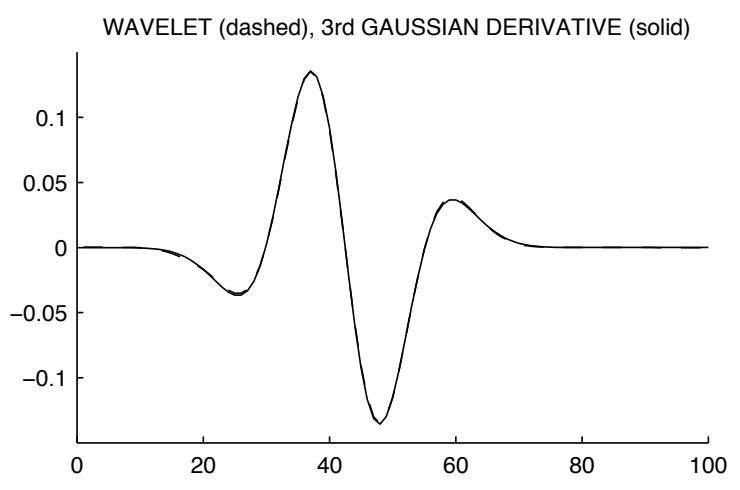

Fig. 14. Example 3 - The discrete-time wavelets are very similar to the 3rd derivative of the Gaussian function. The plot illustrates one of the discrete-time wavelets (dashed) and the the 3rd derivative of the Gaussian with parameters chosen to match (solid).

is dense, the proposed overcomplete rational DWT may provide a fast, efficient alternative to the Mexicanhat-based continuous wavelet transform. Compared to the continuous wavelet transform, the overcomplete rational DWT is (a) efficient to compute because it is implemented by iterating a discrete-time FIR filter bank, (b) less redundant, and (c) exactly and efficiently invertible.

Applications, properties, and advantages of the complex (analytic) Mexican hat wavelet are described in [24]. In [24], a generalization of the complex Mexican hat wavelet is introduced and shown to have excellent properties, especially for the measurement of the instantaneous amplitude and frequency of oscillating signals. Therefore, the investigation of complex overcomplete rational discrete wavelet transforms, for example, using the dual-tree approach [27], may also be of interest.

Example 3: In this example we construct an overcomplete rational DWT with three vanishing moments by using the low-pass filter in (32). The least asymmetric, approximately shift-invariant completion of the filter bank is tabulated in Table III. As in Examples 1 and 2, the wavelets closely resemble a derivative of the Gaussian function, as illustrated in Fig. 14.

Example 4 (Denoising): In order to evaluate the proposed overcomplete rational DWT, we illustrate its use for 1-D signal denoising. We use the 'Bumps' signal of length 1000 from the WAVELAB package [19] (normalized so that the maximum value is 1 ) and iid Gaussian noise with $\sigma=0.1$. For this signal, we perform denoising via hard thresholding utilizing the DWT, undecimated DWT (UDWT), orthonormal rational DWT, and the overcomplete rational DWT. For the dyadic transforms, we use Daubechies filters with 3 vanishing moments. For the orthonormal rational DWT, we use the minimal-length filters with 3 vanishing moments, tabulated in [5]. For the overcomplete rational DWT, we use the filters in Example 3, 
TABLE III

EXAMPLE 3 - THE HIGH-PASS FILTERS $g_{i}(n)$ COMPLETING THE LOW-PASS FILTER (32) TO AN OVERCOMPLETE RATIONAL FILTER BANK. ONE OF THE WAVELETS IS ILLUSTRATED IN FIG. 14.

\begin{tabular}{rrrr}
\hline$n$ & $g_{0}(n)$ & $g_{1}(n)$ & $g_{2}(n)$ \\
\hline 0 & 0.27233206479977 & 0 & 0 \\
1 & -0.61908076926010 & 0.26836075046404 & 0 \\
2 & 0.28915412481143 & -0.59762157010140 & 0.26525899687917 \\
3 & 0.13628795962451 & 0.25097019745787 & -0.59690708656455 \\
4 & -0.03722408597653 & 0.16048269813665 & 0.27076454631514 \\
5 & -0.03033086936698 & -0.03531879266556 & 0.12808768533827 \\
6 & -0.01045600463704 & -0.03789399359700 & -0.01779557105036 \\
7 & -0.00065471929249 & -0.00783324728581 & -0.03958052448987 \\
8 & -0.00002770070659 & -0.00114604240716 & -0.00903314288243 \\
9 & 0 & 0 & -0.00075793021949 \\
10 & 0 & 0 & -0.00003697332591 \\
\hline
\end{tabular}

tabulated in Table III. The dyadic transforms are iterated for four stages whereas the rational transforms are iterated for seven stages. We make this choice so that, for the dyadic and rational transforms, the subband signal at the lowest resolution level are comparable in length, because $(2 / 3)^{7} \approx(1 / 2)^{4}$. Varying the threshold and using 100 noise realizations, we obtain the average RMSE curves given in Fig. 15. Even though the overcomplete rational DWT is less redundant than the UDWT (three-times instead of five-times redundant) its performance is superior for well chosen thresholds, at least for this example.

The wavelets used in Fig. 15 are short filters having 3 vanishing moments; but we also compare with rational wavelets already constructed for the critically-sampled case [4], [9]. The orthonormal rational DWT implemented using the wavelets of [4] (which are ideal filters that we implement using the FFT) give higher RMSE than the orthonormal dyadic DWT. The orthonormal rational DWT implemented using the rational wavelets of [9] do about the same as or slightly better than the orthonormal dyadic DWT (better than the orthonormal rational DWT in Fig. 15, but not as good as either the undecimated dyadic DWT or overcomplete rational DWT). The result confirms the performance improvement achievable with overcomplete transforms. 


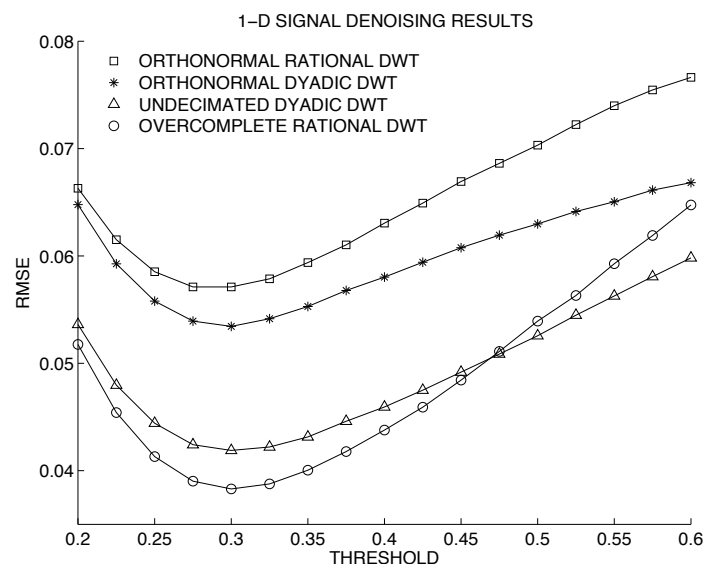

Fig. 15. Denoising using dyadic and rational transforms, orthonormal and overcomplete.

\section{The Q FACTOR OF AN IDEAL OVERCOMPlETE RATIONAL WAVELET TrANSFORM}

Because the band-pass filters in a dyadic wavelet transform have low Q factors, the dyadic WT is not suitable for a number of applications that require higher Q factors [12], [8], [18]. One attraction of rational wavelet transforms is, therefore, the higher Q factor of the band-pass filters. In this section, we investigate the $\mathrm{Q}$ factors of the overcomplete rational filter bank. To simplify the analysis we assume the dilation factor is $3 / 2$ and that the filters are ideal brick-wall filters.

If $1 / 6<a<1 / 3$, then the ideal low-pass and high-pass filters

$$
\begin{aligned}
& H(\omega)= \begin{cases}\sqrt{6} & \text { if }|\omega| \leq a \pi, \\
0 & \text { if } a \pi<|\omega| \leq \pi,\end{cases} \\
& G(\omega)= \begin{cases}0 & \text { if }|\omega| \leq 2 a \pi, \\
1 & \text { if } 2 a \pi<|\omega| \leq \pi,\end{cases}
\end{aligned}
$$

are perfect reconstruction filters for the partially shift-invariant filter bank illustrated in Fig. 7. (The filters satisfy $(5 a),(5 b)$, and $(5 c)$.

Let us compute the $\mathrm{Q}$ factors of the band-pass filter at each level of the partially shift-invariant rational DWT based on iterating the filter bank in Fig. 7. With the filters in (47), the resonant frequency (defined to be the geometric mean of the band edges) and the bandwidth of the first level (that is, of the high-pass filter $G(\omega))$ is

$$
\mathrm{RF}_{1}=\sqrt{2 a} \pi, \quad \mathrm{BW}_{1}=(1-2 a) \pi,
$$


so that the $\mathrm{Q}$ factor (defined to be the ratio of the resonant frequency to the bandwidth) at the finest scale is

$$
\mathrm{Q}_{1}=\frac{\sqrt{2 a}}{1-2 a} .
$$

It can be verified that the filter response at level $j$ is, for $j>1$,

$$
G^{(j)}(\omega)= \begin{cases}\left(\frac{3}{2}\right)^{(j-1) / 2} & \text { if } 2\left(\frac{2}{3}\right)^{j} a \pi<|\omega| \leq 3\left(\frac{2}{3}\right)^{j} a \pi \\ 0 & \text { for other }|\omega| \leq \pi\end{cases}
$$

Hence, the resonant frequency and the bandwidth at level $j$ is

$$
\mathrm{RF}_{j}=\sqrt{6}\left(\frac{2}{3}\right)^{j} a \pi, \quad \mathrm{BW}_{j}=\left(\frac{2}{3}\right)^{j} a \pi,
$$

so that the $\mathrm{Q}$ factor at scale $j>1$ becomes $\mathrm{Q}_{j}=\sqrt{6}$. Therefore, the overcomplete rational DWT provides a constant $\mathrm{Q}$ analysis for all scales, except the finest scale.

Note that if $a=1 / 3$, then the filters in (47) (multiplied by $\sqrt{3}$ ) are PR filters for an orthonormal rational filter bank. The $\mathrm{Q}$ factor for the orthonormal case is $\mathrm{Q}_{j}^{\text {orth }}=\sqrt{6}$ for $j \geq 1$. Hence the constant $\mathrm{Q}$ property for the orthonormal case. We remark that the dyadic DWT with ideal (sinc) filters has a Q-factor equal to $\sqrt{2}$. Thus the rational DWT provides a constant-Q transform with a higher $\mathrm{Q}$.

\section{REMARKS}

Degree of Redundancy. The proposed approach for the design of overcomplete rational filter banks requires a specific degree of redundancy. For example, if the dilation factor is $3 / 2$, then the filter bank must be $5 / 3$-times overcomplete, as in Fig. 8. In general, if the dilation factor is $q / p$, then the filter bank must be $(q+p) / p$-times overcomplete. Although overcomplete rational filter banks can be designed with other redundancy ratios, they can not be obtained using the approach described in this paper. In order to perform the filter bank completion using the matrix spectral factorization approach, it is important that the matrix $\mathbf{G}(z)$ in (36) be square. This in turn, requires that the number of unknown channels be equal to the down-sampling factor. For example, if the overcomplete rational filter bank in Fig. 8 had two $G_{i}(z)$ channels instead of three, then the matrix $\mathbf{G}(z)$ would not be square and the completion of the filter bank given the low-pass filter $H(z)$ would not constitute a matrix spectral factorization problem. In this case, other methods must be employed for the filter bank completion step.

Therefore, the effectiveness of the proposed approach is limited to a specific redundancy ratio (dependent on the rational dilation factor). Note that this redundancy ratio is the one that corresponds to the partially shift-invariant overcomplete rational filter bank illustrated in Fig. 7. Hence, the proposed 
approach can be used to design approximately shift-invariant rational DWTs based on any rational dilation factor $q / p$.

Other Rational Dilation Factors. Although the proposed approach has been illustrated and described for the dilation factor $3 / 2$, this approach can be used for other rational dilation factors as well. If the dilation factor is $q / p$ instead of $3 / 2$, then the filter bank illustrated in Fig. 8 will have $q$ high-pass $G_{i}(z)$ channels instead of three. Similarly, the filter bank illustrated in Fig. 9 will have $p$ low-pass $H_{i}(z)$ channels instead of two. The overcomplete rational filter bank will then be $(q+p) / p$-times overcomplete. Additionally, note that the discrete wavelet transform implemented by iterating the filter bank will be $p /(p-q)$-times overcomplete.

In order to obtain a higher density time-frequency lattice, so that the overcomplete rational DWT transform well approximates the continuous wavelet transform, one may use the dilation factor $p /(p-1)$ with larger $p$ (say $p>3$ ), at the cost of increased redundancy as discussed above.

\section{CONCLUSION}

This paper illustrates that overcomplete discrete wavelet transforms based on rational dilation factors are free of some of the problems of critically-sampled rational DWTs. Specifically:

1) The proposed overcomplete rational DWT is approximately shift-invariant, in contrast to the criticallysampled rational DWT which is even more shift-varying than the critically-sampled dyadic DWT.

2) Minimal-length perfect reconstruction filters for the overcomplete rational DWT can be straightforwardly designed to have a specified number of vanishing moments. For the orthonormal rational DWT, no such construction is known.

3) The analysis/synthesis functions (discrete-time wavelets) can be very smooth and can be designed so as to closely resemble the derivatives of the Gaussian function.

4) Due to the higher density of time-frequency lattice, the proposed overcomplete rational DWT can provide an efficient, exactly invertible filter bank implementation of the continuous wavelet transform.

This paper complements other work on the construction of overcomplete wavelet transforms (frames), much of which is concerned with the dyadic case. Compared with the dyadic DWT, the rational DWT provides a more gradual change in scale, from one scale to the next. Therefore, in principle, it may be more likely that there exist analysis/synthesis functions that are well matched to specific signal features. 


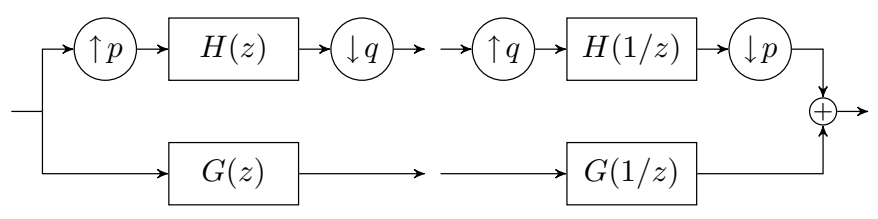

Fig. 16. Partially shift-invariant overcomplete rational filter bank. It attains the time-frequency lattice illustrated in the bottom panel of Fig. 2. However, this filter bank perfect reconstruction with useful FIR filters.

\section{ACKNOWLEDGMENT}

The authors thank Bin Han, University of Alberta, for comments regarding the filter bank completion problem.

\section{APPENDIX}

In this appendix, we show that the FB in Fig. 16 does not admit perfect reconstruction when the filters' transfer functions are rational functions of $z$. This negative result was shown in [38] for the case ( $p=1$, $q=2)$. Here we show it for the more general case $(p<q)$.

Let us denote the polyphase components of $H(z)$ by $H_{i}(z), i=0,1, \ldots, p-1$, which are defined through

$$
H(z)=\sum_{i=0}^{p-1} z^{-q i} H_{i}\left(z^{p}\right) .
$$

Then, the PR condition is equivalent to,

$$
\left[\begin{array}{ccccc}
H_{0}(z) & H_{1}(z) & \ldots & H_{p-1}(z) & q G(z) \\
H_{0}\left(z W_{q}\right) & H_{1}\left(z W_{q}\right) & \ldots & H_{p-1}\left(z W_{q}\right) & 0 \\
\vdots & & \ddots & & \vdots \\
H_{0}\left(z W_{q}^{q-1}\right) & H_{1}\left(z W_{q}^{q-1}\right) & \ldots & H_{p-1}\left(z W_{q}^{q-1}\right) & 0
\end{array}\right] \cdot\left[\begin{array}{c}
H_{0}(1 / z) \\
H_{1}(1 / z) \\
\vdots \\
H_{p-1}(1 / z) \\
G(1 / z)
\end{array}\right]=\left[\begin{array}{c}
q \\
0 \\
\vdots \\
0 \\
0
\end{array}\right]
$$

where $W_{q}=e^{j 2 \pi / q}$. Considering the lower left $p \times p$ portion of the matrix on the left hand side, we have the following lemma. 
Lemma 1: If $H_{i}(z)$ are rational functions and

$$
\underbrace{\left[\begin{array}{ccc}
H_{0}\left(z W_{q}^{q-p}\right) & \ldots & H_{p-1}\left(z W_{q}^{q-p}\right) \\
\vdots & \ddots & \vdots \\
H_{0}\left(z W_{q}^{q-1}\right) & \ldots & H_{p-1}\left(z W_{q}^{q-1}\right)
\end{array}\right]}_{\mathbf{H}_{\mathbf{m}}(z)} \underbrace{\left[\begin{array}{c}
H_{0}(1 / z) \\
H_{1}(1 / z) \\
\vdots \\
H_{p-1}(1 / z)
\end{array}\right]}_{\mathbf{H}_{\mathbf{r}}(z)}=\mathbf{0},
$$

then $\mathbf{H}_{\mathbf{m}}(z)$ is not full normal rank (i.e. its determinant is zero).

Proof: Assume that $\mathbf{H}_{\mathbf{m}}(z)$ is full normal rank so that its determinant is a rational function of $z$, denoted by $d(z)$. Since $H_{i}(z)$ are rational functions, $d(z)$ has a finite set of zeros and poles, $R_{d}=\left\{z_{n}\right\}_{n}$. Also let $B_{i}$ denote the set of zeros and poles of $H_{i}(1 / z)$. Now pick $z^{*} \notin\left(R_{d} \cup\left(\cup_{i} B_{i}\right)\right)$. Then $\mathbf{H}_{\mathbf{m}}\left(z^{*}\right)$ is full rank and $\mathbf{H}_{\mathbf{r}}\left(z^{*}\right) \neq \mathbf{0}$. Consequently we have $\mathbf{H}_{\mathbf{m}}\left(z^{*}\right) \mathbf{H}_{\mathbf{r}}\left(z^{*}\right) \neq \mathbf{0}$, which contradicts (52).

Corollary 1: If $H_{i}(z)$ are rational functions and (52) holds, then

$$
\left[\begin{array}{c}
H_{0}(z) \\
H_{1}(z) \\
\vdots \\
H_{p-1}(z)
\end{array}\right]^{T}=\left[\begin{array}{c}
c_{0}(z) \\
c_{1}(z) \\
\vdots \\
c_{p-2}(z)
\end{array}\right]^{T}\left[\begin{array}{ccc}
H_{0}\left(z W_{q}^{q-p+1}\right) & \ldots & H_{p-1}\left(z W_{q}^{q-p+1}\right) \\
\vdots & \ddots & \vdots \\
H_{0}\left(z W_{q}^{q-1}\right) & \ldots & H_{p-1}\left(z W_{q}^{q-1}\right)
\end{array}\right]
$$

where $c_{i}(z)$ are rational functions.

The corollary follows by modulating $\mathbf{H}_{\mathbf{m}}(z)$ and using Lemma 1. As a corollary of this corollary, we reach the desired negative result.

Corollary 2: If $H_{i}(z)$ are rational functions, then (51) holds iff $H_{i}(z)=0$ and $G(z)$ is allpass.

Proof: Sufficiency is obvious. To see necessity, note that (51) implies (52). Using the corollary, we have

$\left[\begin{array}{c}H_{0}(z) \\ H_{1}(z) \\ \vdots \\ H_{p-1}(z)\end{array}\right]^{T}\left[\begin{array}{c}H_{0}(1 / z) \\ H_{1}(1 / z) \\ \vdots \\ H_{p-1}(1 / z)\end{array}\right]=\left[\begin{array}{c}c_{0}(z) \\ c_{1}(z) \\ \vdots \\ c_{p-2}(z)\end{array}\right]^{T}\left[\begin{array}{clc}H_{0}\left(z W_{q}^{q-p+1}\right) & \ldots & H_{p-1}\left(z W_{q}^{q-p+1}\right) \\ \vdots & \ddots & \vdots \\ H_{0}\left(z W_{q}^{q-1}\right) & \ldots & H_{p-1}\left(z W_{q}^{q-1}\right)\end{array}\right]\left[\begin{array}{c}H_{0}(1 / z) \\ H_{1}(1 / z) \\ \vdots \\ H_{p-1}(1 / z)\end{array}\right]=\mathbf{0}$,

from which it follows that $H_{i}(z)=0$. Eqn. (51) implies that $G(z)$ should be allpass.

\section{REFERENCES}

[1] P. Addison. The Illustrated Wavelet Transform Handbook. Taylor and Francis, 2002. 
[2] F. Argenti and E. Del Re. Rational sampling filter banks based on IIR filters. IEEE Trans. Signal Processing, 46(12):34033408, December 1998.

[3] P. Auscher. Wavelet bases for $L^{2}(R)$ with rational dilation factor. In M. B. Ruskai et al., editor, Wavelets and Their Applications. Jones and Barlett, Boston, 1992.

[4] A. Baussard, F. Nicolier, and F. Truchetet. Rational multiresolution analysis and fast wavelet transform: application to wavelet shrinkage denoising. Signal Processing, 84(10):1735-1747, October 2004.

[5] İ. Bayram and I. Selesnick. Design of orthonormal and overcomplete wavelet transforms based on rational sampling factors. In Proc. Fifth SPIE Conference on Wavelet Applications in Industrial Processing, 2007.

[6] P. Blanc, T. Blu, T. Ranchin, L. Wald, and R. Aloisi. Using iterated rational filter banks within the ARSIS concept for producing 10m Landsat multispectral images. Int. J. of Remote Sensing, 19(12):2331-2343, August 1998.

[7] T. Blu. Iterated filter banks with rational rate changes — connection with discrete wavelet transforms. IEEE Trans. Signal Processing, 41(12):3232-3244, December 1993.

[8] T. Blu. An iterated rational filter bank for audio coding. In Proc. IEEE Int. Symposium on Time-frequency and Time-scale Analysis, 1996.

[9] T. Blu. A new design algorithm for two-band orthonormal rational filter banks and orthonormal rational wavelets. IEEE Trans. Signal Processing, 46(6):1494-1504, June 1998.

[10] P. J. Burt and E. H. Adelson. The Laplacian pyramid as a compact image code. IEEE Trans. Comm., 31(4):532-540, April 1983.

[11] F. Callier. On polynomial matrix spectral factorization by symmetric extraction. IEEE Trans. Automatic Control, 30(5):453464, May 1985.

[12] G. F. Choueiter and J. R. Glass. An implementation of rational wavelets and filter design for phonetic classification. IEEE Trans. Audio, Speech and Language Processing, 15(3):939-948, March 2007.

[13] C. Chui and W. He. Compactly supported tight frames associated with refinable functions. J. of Appl. and Comp. Harm. Analysis, 8(3):293-319, May 2000.

[14] C. K. Chui, W. He, and J. Stöckler. Compactly supported tight and sibling frames with maximum vanishing moments. J. of Appl. and Comp. Harm. Analysis, 13(3):224-262, November 2002.

[15] T. Cooklev, A. Nishihara, M. Kato, and M. Sablatash. Two-channel multifilter banks and multiwavelets. In Proc. IEEE Int. Conf. Acoust., Speech, Signal Processing (ICASSP), 1996.

[16] I. Daubechies. Ten Lectures on Wavelets. SIAM, 1992.

[17] M. Davis. Factoring the spectral matrix. IEEE Trans. Automatic Control, 8(4):296-305, October 1963.

[18] F. C. C. B. Diniz, I. Koethe, S. L. Netto, and L. W. P. Biscainho. High-selectivity filter banks for spectral analysis of music signals. EURASIP Journal on Advences in Signal Processing, 2007:Article ID 94704, 12 pages, 2007. doi:10.1155/2007/94704.

[19] D. Donoho, A. Maleki, and M. Shahram. Wavelab 850. Technical report, Stanford University, 2005. http://www-stat. stanford.edu/ $\sim$ wavelab/.

[20] K. Egiazarian and J. Astola. Tree-structured Haar transforms. J. Mathematical Imaging and Vision, 16(3):269-279, May 2002.

[21] L. Ephremidze, G. Janashia, and E. Lagvilava. A new efficient matrix spectral factorization algorithm. In Proc. SICE Annual Conference, pages 29-38, Kagawa University, Japan, September 17-20 2007. 
[22] B. Han and Q. Mo. Splitting a matrix of Laurent polynomials with symmetry and its application to symmetric framelet filter banks. SIAM Journal on Matrix Analysis and its Appliations, 26(1):97-124, 2004.

[23] D. P. Hardin, T. A. Hogan, and Q. Sun. The matrix-valued Riesz lemma and local orthonormal bases in shift-invariant spaces. Adv. Comput. Math., 20(4):367-384, September 2004.

[24] Jonathan D. Harrop. Structural Properties of Amorphous Materials. PhD thesis, University of Cambridge, 2004.

[25] M. Holschneider, R. Kronland-Martinet, J. Morlet, and A. Grossmann. A real-time algorithm for signal analysis with the help of the wavelet transform. In J. M. Combes, A. Grossmann, and Ph. Tchamitchian, editors, Wavelets, Time-Frequency Methods and Phase Space, pages 286-297. Springer-Verlag, 1989.

[26] B. D. Johnson. Stable filtering schemes with rational dilations. J. Fourier Anal. App., 13(5):607-621, September 2007.

[27] N. G. Kingsbury. Complex wavelets for shift invariant analysis and filtering of signals. J. of Appl. and Comp. Harm. Analysis, 10(3):234-253, May 2001.

[28] J. Kovačević and A. Chebira. Life beyond bases: The advent of frames (Part II). IEEE Signal Processing Magazine, 24(5):115-1125, September 2007.

[29] J. Kovačević and M. Vetterli. Perfect reconstruction filter banks with rational sampling factors. IEEE Trans. Signal Processing, 41(6):2047-2066, June 1993.

[30] V. Kučera. Analysis and Design of Discrete Linear Control Systems. Prentice Hall, 1991.

[31] V. Kučera. Factorization of rational spectral matrices: A survey of methods. In Proc. IEE Int. Conf. on Control, 1991.

[32] J. Lebrun and I. Selesnick. Gröbner bases and wavelet design. Journal of Symbolic Computing, 37(2):227-259, February 2004.

[33] D. Marr and E. Hildreth. Theory of edge detection. Proceedings of the Royal Society of London, B207(1167):187-217, February 1990.

[34] P. Motyka and J. Cadzow. The factorization of discrete-process spectral matrices. IEEE Trans. Automatic Control, 12(6):698-707, December 1967.

[35] S. U. Pillai and T. I. Shim. Spectrum Estimation and System Identification. Springer-Verlag, 1993.

[36] O. Rioul and T. Blu. Simple regularity criteria for subdivision schemes. II. The rational case, $1997 . \quad$ citeseer.ifi.uzh.ch/rioul97simple.html.

[37] A. Ron and Z. Shen. Construction of compactly supported affine frames in $L_{2}\left(\mathbb{R}^{d}\right)$. In K. S. Lau, editor, Advances in Wavelets. Springer-Verlag, 1998.

[38] I. W. Selesnick. The double density DWT. In A. Petrosian and F. G. Meyer, editors, Wavelets in Signal and Image Analysis: From Theory to Practice. Kluwer, 2001.

[39] I. W. Selesnick. A higher-density discrete wavelet transform. IEEE Trans. Signal Processing, 54(8):3039-3048, 2006.

[40] E. P. Simoncelli, W. T. Freeman, E. H. Adelson, and D. J. Heeger. Shiftable multi-scale transforms. IEEE Trans. Information Theory, 38(2):587-607, March 1992.

[41] M. J. T. Smith and T. P. Barnwell III. Exact reconstruction for tree-structured subband coders. IEEE Trans. Acoust., Speech, and Signal Proc., 34(3):431-441, June 1986.

[42] P. Steffen, P. N. Heller, R. A. Gopinath, and C. S. Burrus. Theory of regular M-band wavelet bases. IEEE Trans. Signal Processing, 41(12):3497-3511, December 1993.

[43] G. Strang. Linear Algebra and its Applications. Brooks Cole, $3^{\text {rd }}$ edition, 1988.

[44] P. P. Vaidyanathan. Multirate Systems and Filter Banks. Prentice Hall, 1992. 
[45] M. Vetterli and J. Kovačević. Wavelets and Subband Coding. Prentice Hall, $1995 . \quad$ Online at http://www.waveletsandsubbandcoding.org/.

[46] D. Youla. On the factorization of rational matrices. IEEE Trans. Information Theory, 7(3):172-189, July 1961. 